\title{
A Bayesian model to correct underestimated 3-D wind speeds from sonic anemometers increases turbulent components of the surface energy balance
}

\author{
John M. Frank ${ }^{1,2}$, William J. Massman ${ }^{1}$, and Brent E. Ewers ${ }^{2}$ \\ ${ }^{1}$ US Forest Service, Rocky Mountain Research Station, 240 W. Prospect Rd., Fort Collins, CO 80526, USA \\ ${ }^{2}$ University of Wyoming, Department of Botany and Program in Ecology, 1000 E. University Ave, Laramie, WY 82071, USA \\ Correspondence to: John M. Frank (jfrank@fs.fed.us)
}

Received: 25 April 2016 - Published in Atmos. Meas. Tech. Discuss.: 23 June 2016

Revised: 17 November 2016 - Accepted: 21 November 2016 - Published: 12 December 2016

\begin{abstract}
Sonic anemometers are the principal instruments in micrometeorological studies of turbulence and ecosystem fluxes. Common designs underestimate vertical wind measurements because they lack a correction for transducer shadowing, with no consensus on a suitable correction. We reanalyze a subset of data collected during field experiments in 2011 and 2013 featuring two or four CSAT3 sonic anemometers. We introduce a Bayesian analysis to resolve the threedimensional correction by optimizing differences between anemometers mounted both vertically and horizontally. A grid of 512 points ( $\sim \pm 5^{\circ}$ resolution in wind location) is defined on a sphere around the sonic anemometer, from which the shadow correction for each transducer pair is derived from a set of 138 unique state variables describing the quadrants and borders. Using the Markov chain Monte Carlo (MCMC) method, the Bayesian model proposes new values for each state variable, recalculates the fast-response data set, summarizes the $5 \mathrm{~min}$ wind statistics, and accepts the proposed new values based on the probability that they make measurements from vertical and horizontal anemometers more equivalent. MCMC chains were constructed for three different prior distributions describing the state variables: no shadow correction, the Kaimal correction for transducer shadowing, and double the Kaimal correction, all initialized with $10 \%$ uncertainty. The final posterior correction did not depend on the prior distribution and revealed both self- and cross-shadowing effects from all transducers. After correction, the vertical wind velocity and sensible heat flux increased $\sim 10 \%$ with $\sim 2 \%$ uncertainty, which was significantly higher than the Kaimal correction. We applied
\end{abstract}

the posterior correction to eddy-covariance data from various sites across North America and found that the turbulent components of the energy balance (sensible plus latent heat flux) increased on average between 8 and $12 \%$, with an average $95 \%$ credible interval between 6 and $14 \%$. Considering this is the most common sonic anemometer in the AmeriFlux network and is found widely within FLUXNET, these results provide a mechanistic explanation for much of the energy imbalance at these sites where all terrestrial/atmospheric fluxes of mass and energy are likely underestimated.

\section{Copyright statement}

This paper was written and prepared by a US Government employee on official time, and therefore it is in the public domain and not subject to copyright.

\section{Introduction}

The eddy-covariance technique has become the most commonly used method for measuring the ecosystem exchange of mass and energy with the atmosphere. It is fundamental to the global network of flux towers that are central to quantifying terrestrial carbon sinks and sources (Baldocchi, 2003), to hydrological studies accounting for evapotranspiration and sublimation (Biederman et al., 2014; Reba et al., 2012), and to the energy balance through the turbulent fluxes of sensible and latent heat (Welch et al., 2015; Anderson and Wang, 2014). There is a growing consensus within the mi- 


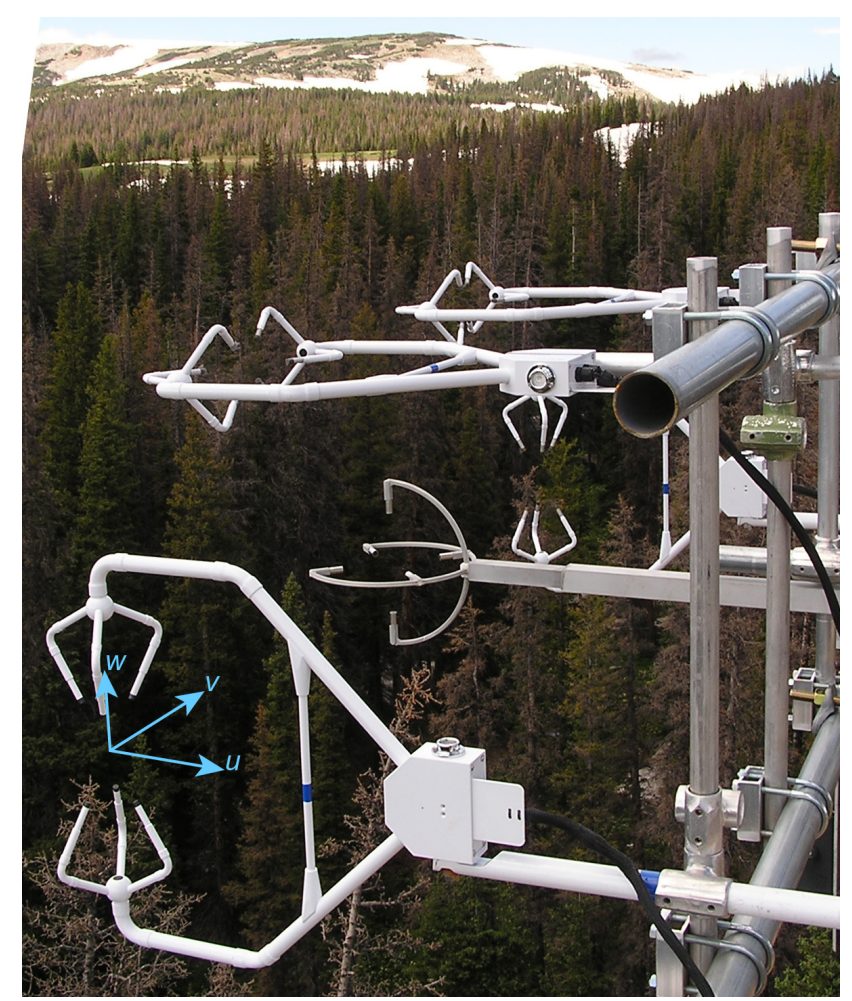

Figure 1. Photograph of the 2011 experiment with two CSAT3 sonic anemometers mounted vertically and two horizontally. The cardinal $\boldsymbol{u}, \boldsymbol{v}$, and $\boldsymbol{w}$ axes are shown in light blue near one of the vertical instruments. Figure from Frank et al. (2013).

crometeorology and ecosystem flux communities that many sonic anemometers, the core instrument for all modern eddycovariance systems, systematically underestimate the vertical wind component (Frank et al., 2016; Horst et al., 2015; Kochendorfer et al., 2012). The ramifications for this are that all vertical fluxes (i.e., carbon dioxide, water vapor, latent heat, sensible heat, momentum) are similarly underestimated for any ecosystem. This underestimate is roughly consistent with the persistent energy balance closure problem across flux sites (Leuning et al., 2012; Stoy et al., 2013; Wilson et al., 2002), where a vast majority are assumed to be systematic biased towards low turbulent fluxes of sensible and latent heat.

Horst et al. (2015) and Frank et al. (2016) have shown that the error in at least two non-orthogonal sonic anemometer designs can be traced to transducer shadowing that remains uncorrected in the anemometer's firmware. In both studies, shadowing was described a priori by theoretical formulations based on the wind tunnel tests of Kaimal (1979), yet there was no consensus on a correction. A shortcoming in the use of formulations derived for single transducer pairs in laminar flow to describe turbulent flow distortions around more complex geometries (Fig. 1) is that shadowing between all transducers and structures cannot be accurately represented or incorporated. A second problem is that in turbulent flow fields there are few standards available to use as a calibration reference. Advancements in Bayesian techniques (Gelman et al., 2014) have created the potential to resolve both of these issues by incorporating prior knowledge of transducer flow distortions with a model that evaluates the omnidirectionality of a sonic anemometer to produce a posterior 3-D correction.

To quantify a 3-D correction of the CSAT3 sonic anemometer, we reanalyze data from field experiments conducted by Frank et al. (2013, 2016), where wind measurements from non-orthogonal anemometers mounted vertically and horizontally were significantly different. We develop a Bayesian hierarchical model to evaluate three hypotheses:

1. A 3-D shadowing correction based solely on wind location can make a non-orthogonal sonic anemometer omnidirectional.

2. This correction increases vertical wind measurements more than expected from single transducer shadowing because it accurately represents all shadowing between transducers.

3. In ecosystems where these instruments are deployed, the application of this correction will result in significantly higher Bayesian credible intervals for the turbulent components of the energy budget and improved surface energy budget closure.

\section{Methods}

\subsection{Reanalysis of field experiments}

We reanalyze data from field campaigns conducted by Frank et al. (2013, 2016). To summarize them, experiments were conducted in 2011 and 2013 where multiple sonic anemometers were deployed in a horizontal array at $24.5 \mathrm{~m}$ height on the Glacier Lakes Ecosystem Experiments Site (GLEES) AmeriFlux scaffold above a subalpine forest in southeastern Wyoming, USA (Frank et al., 2014). The anemometers were initially mounted vertically, oriented west, arranged south to north, staggered up and down, and located $0.50 \mathrm{~m}$ center-to-center from each other (Fig. 1). Periodically, some of the anemometers were rotated $90^{\circ}$ around their $\boldsymbol{u}$ axis and mounted horizontally. In this study we focus only on the CSAT3 sonic anemometer (Campbell Scientific, Inc., Logan, UT, USA) during times when both vertically and horizontally mounted anemometers were present (Table 1). All anemometers were operated with firmware version 4.0. It is conventional to describe the three dimensions of a sonic anemometer as the $\boldsymbol{u}, \boldsymbol{v}$, and $\boldsymbol{w}$ axes. To reduce confusion in describing horizontal anemometers, we refer to cardinal $\boldsymbol{u}, \boldsymbol{v}$, and $\boldsymbol{w}$, where the measurements have been rotated to west-east $(\boldsymbol{u})$, south-north $(\boldsymbol{v})$, and down-up $(\boldsymbol{w})$, which are consistent with $\boldsymbol{u}, \boldsymbol{v}$, and $\boldsymbol{w}$ for vertically mounted anemometers. 
Table 1. Summary of the subset of data from Frank et al. $(2013,2016)$ reanalyzed in this study listing the four CSAT3 anemometers (A-D), their location within the five-position horizontal array, and whether they are mounted horizontally $(*)$. Because processing the Bayesian model is extremely intensive, only $5 \%$ of the available data were reanalyzed.

\begin{tabular}{llllll|r|rr}
\hline & \multicolumn{5}{c}{ Position } & Number of 5 min periods \\
\cline { 2 - 8 } Dates & 1 & 2 & 3 & 4 & 5 & Available & Reanalyzed \\
\hline 5-19 July 2011 & A $^{*}$ & B & - & C & D* & 2520 & 126 \\
19-26 July 2011 & A & B $^{*}$ & - & C $^{*}$ & D & 1992 & 100 \\
9-16 August 2011 & B $^{*}$ & A & - & D & C & 1974 & 98 \\
16-22 August 2011 & B & A* & - & D $^{*}$ & C & 1620 & 81 \\
26-30 July 2013 & A $^{*}$ & - & B & - & - & 906 & 46 \\
23-27 August 2013 & - & - & A & - & B $^{*}$ & 1050 & 52 \\
6-24 September 2013 & - & - & B & D $^{*}$ & - & 498 & 25 \\
\hline
\end{tabular}

Finally, because our Bayesian model is computationally intensive, we reanalyze a subset of only $5 \%$ of the available data (see Sect. 2.3).

\subsection{The Bayesian model}

Bayesian statistics is based on Bayes' theorem (Bayes and Price, 1763), which in modern applications relates the posterior probability of a model parameter conditioned on data to the product of the likelihood of the data and the prior probability of that parameter (Gelman et al., 2014). In essence, the prior represents an initial educated guess or belief in the value of a model parameter; the likelihood is the probability of observing the data if they were deterministically generated from a model; and the posterior is an updated belief in the model parameter considering each the prior, the model, and the data. Analytical evaluation of the posterior is rarely possible, as is in our case; thus the posterior is commonly estimate through the Markov chain Monte Carlo (MCMC) method, Gibbs sampling (Appendix A1), and the Metropolis-Hastings algorithm (Kruschke, 2010). The framework of our Bayesian model is to divide the sphere around the sonic anemometer into approximately equal grid points and to define a prior probability distribution of the 3D shadowing correction for each transducer pair at each location. Then, the model proposes new corrections for each grid point, recalculates the fast-response data set, summarizes new $5 \mathrm{~min}$ wind statistics, determines the probability that the updated measurements from vertical and horizontal anemometers are more equivalent using the proposed correction versus the old one (i.e., the Metropolis-Hastings ratio, which is Eq. A13 evaluated for the proposed versus old correction), and finally accepts/rejects the proposal probabilistically from this ratio to construct the posterior correction. The model recursively adjusts the distribution that generates the proposals to achieve between 25 and $50 \%$ acceptance rates, which are theoretically optimal (Gelman et al., 2014). We define a grid of 512 points $\left(\sim \pm 5^{\circ}\right.$ resolution of wind location) on a sphere around each of the three transducer pairs of the sonic anemometer. Neglecting the upper and lower mount- ing arms that extend back into the electronics housing and support block, the CSAT3 is symmetrical on either side of a transducer pair, between the upper and lower hemispheres, and for each of the three transducer pairs. To pool data and reduce computations, we make these assumptions of symmetry to describe all 1536 points from a set of 138 unique state variables.

In our mathematical notation, we use uppercase and lowercase subscripts to distinguish variables as scalars, vectors, or matrices. Uppercase subscripts are part of the variable name, denote the dimensionality of the variable, and describe the coordinate system. For example, $\mathbf{M}_{S \times T}$ is a two-dimensional matrix with dimensions $S$ and $T$, which correspond to sonic and transducer coordinates; since there are three dimensions for both coordinate systems, this is a $3 \times 3$ matrix. One uppercase subscript by itself denotes a vector in that coordinate system. Lowercase subscripts denote indexing for variables that are defined for multiple times or replicate anemometers; these are essentially multidimensional arrays. When the same letter appears as both an uppercase and lowercase subscript, this refers to the $c$ th element of dimension $C$.

We test three prior corrections: no shadow correction, the Kaimal correction (Kaimal, 1979; Frank et al., 2016; Horst et al., 2015), and a doubling of the Kaimal correction (Frank et al., 2016). The Kaimal correction is defined as $U_{\mathrm{T}_{t}}=(1-0.16+0.16 \theta / 70) \dot{U}_{\mathrm{T}_{t}}$ for $\theta \leq 70^{\circ}$ and $U_{\mathrm{T}_{t}}=\dot{U}_{\mathrm{T}_{t}}$ for $\theta>70^{\circ}$, where $U_{\mathrm{T}}$ and $U_{\mathrm{T}}$ are the measured and corrected wind velocities in transducer coordinates and $\theta$ is the angle between the wind and the transducer acoustic path, $t$.

The model predicts the standard deviation of the data in cardinal coordinates, $\sigma_{\mathrm{C}}$, which is defined during each $5 \mathrm{~min}$ period, $f$, for each replicate sonic anemometer, $i$ (Fig. 1), from a normal distribution with mean $\hat{\sigma}_{\mathrm{C}}$ and standard deviation $\varepsilon$ (Eq. 1).

$\sigma_{\mathrm{C}_{c, f, i}} \sim N\left(\hat{\sigma}_{\mathrm{C}_{c, f, i}, \varepsilon^{-2}}\right)$

The predicted mean is constructed in several steps. First, the state variable for the 3-D correction in transducer coordinates, $\alpha_{\mathrm{T}}$, is defined for each grid point, $g$. Here it does not 
matter if each grid point is independent or that they linked together through symmetry. $\alpha_{\mathrm{T}}$ is given a normal prior probability distribution with mean equal to the prior correction, $P$, evaluated for each transducer pair for wind blowing through the longitude, $\lambda$, and latitude, $\varphi$, associated with each grid point and with a predefined standard deviation equal to 0.1 , or $\pm 10 \%$ uncertainty (Eq. 2).

$\alpha_{\mathrm{T}_{t, g}} \sim N\left(P(t, g), 0.1^{-2}\right)$

The 3-D correction is applied to every $20 \mathrm{~Hz}$ sample, $j$, of the original measured wind velocity data in transducer coordinates, $U_{\mathrm{T}}$. The nominal predictor variable, $h$, selects the corresponding grid point that occurs with every $20 \mathrm{~Hz}$ sample. The corrected $20 \mathrm{~Hz}$ wind velocity in transducer coordinates is $U_{\mathrm{T}}$ (Eq. 3).

$\dot{U}_{\mathrm{T}_{f, i, j}}=U_{\mathrm{T}_{f, i, j}} \cdot \alpha_{\mathrm{T}_{h}}$

The non-orthogonal data are transformed via matrix multiplication into orthogonal sonic coordinates, $U_{\mathrm{S}}$ (Eq. 4).

$\dot{U}_{\mathrm{S}_{f, i, j}}=\mathbf{M}_{S \times T} \dot{U}_{\mathrm{T}_{f, i, j}}$

The matrix, $\mathbf{M}_{S \times T}$, is specific to the CSAT3 geometry (Eq. 5).

$$
\begin{aligned}
\mathbf{M}_{S \times T}= & {\left[\begin{array}{ccc}
-\frac{4}{3} & \frac{2}{3} & \frac{2}{3} \\
0 & \frac{2}{\sqrt{3}} & -\frac{2}{\sqrt{3}} \\
\frac{2}{3 \sqrt{3}} & \frac{2}{3 \sqrt{3}} & \frac{2}{3 \sqrt{3}}
\end{array}\right] } \\
= & {\left[\begin{array}{rrr}
-1.333 & 0.667 & 0.667 \\
0 & 1.155 & -1.155 \\
0.385 & 0.385 & 0.385
\end{array}\right] }
\end{aligned}
$$

For the model to predict data simultaneously from both vertical and horizontal anemometers, a final corrected time series data set is produced in cardinal coordinates, $U_{\mathrm{C}}$

$\dot{U}_{\mathrm{C}_{f, i, j}}=\mathbf{N}_{C \times S_{o}} \dot{U}_{\mathbf{S}_{f, i, j}}$

The matrix $\mathbf{N}_{C \times S}$ is straightforward (Eq. 7), and the nominal predictor variable, $o$, selects the orientation of every $20 \mathrm{~Hz}$ sample.

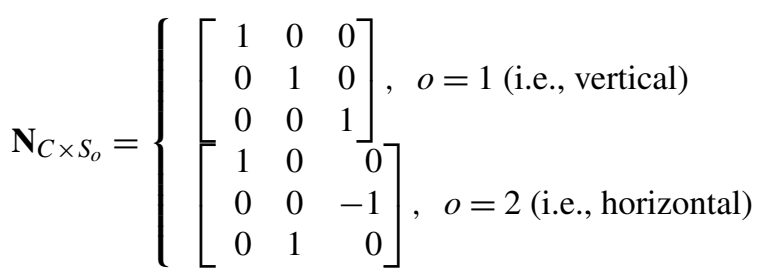

Using the corrected time series data in cardinal coordinates, the model calculates the average correction along the three dimensions, $\beta_{\mathrm{C}}$, for the 5 min standard deviation data for each anemometer (Eq. 8).

$\beta_{\mathrm{C}_{f, i}}=\frac{\sqrt{\frac{1}{J-1} \sum_{j=1}^{J}\left(\dot{U}_{\mathrm{C}_{f, i, j}}-\frac{1}{J} \sum_{j=1}^{J} \dot{U}_{\mathrm{C}_{f, i, j}}\right)^{2}}}{\sqrt{\frac{1}{J-1} \sum_{j=1}^{J}\left(U_{\mathrm{C}_{f, i, j}}-\frac{1}{J} \sum_{j=1}^{J} U_{\mathrm{C}_{f, i, j}}\right)^{2}}}$

Equation (8) is equivalent to the ratio of the standard deviation of $U_{\mathrm{C}}$ divided by the standard deviation of $U_{\mathrm{C}}$, evaluated during each $5 \mathrm{~min}$ period for each sonic anemometer. The reference condition for every $5 \mathrm{~min}$ period, $\widetilde{\sigma}_{\mathrm{C}}$, is a state variable representing the "true" standard deviation of wind velocity. It is assigned a uniform prior probability distribution that generously includes the true value by allowing each $\widetilde{\sigma}_{\mathrm{C}}$ to range from 0 to the maximum of all $U_{\mathrm{C}}$ measurements (Eq. 9).

$\widetilde{\sigma}_{\mathrm{C}_{c, f}} \sim \operatorname{Unif}\left(0, \max \left(U_{\mathrm{C}}\right)\right)$

Finally, the model predicts the mean for the standard deviation data as the reference divided by the average correction (Eq. 10).

$\hat{\sigma}_{\mathrm{C}_{f, i}}=\frac{\widetilde{\sigma}_{\mathrm{C}_{f}}}{\beta_{\mathrm{C}_{f, i}}}$

To complete the Bayesian model definition, the model error is a state variable which is assigned a prior probability distribution with a gamma distribution (Eq. 11).

$\varepsilon \sim \Gamma(1, \hat{b})$

The variance of the gamma distribution, $\hat{b}$, is assigned the same variance as the prior distribution for $\widetilde{\sigma}_{\mathrm{C}}$ which is a uniform distribution (Eq. 12).

$\dot{b}=\frac{\sqrt{12}}{\max \left(U_{\mathrm{C}}\right)-0}$

Distributions are defined where normal distributions are $\theta \sim N(a, b)$ with expected value $E(\theta)=a$ and variance $\operatorname{var}(\theta)=1 / b^{2}$, gamma distributions are $\theta \sim \Gamma(a, b)$ with $E(\theta)=a / b$ and $\operatorname{var}(\theta)=a / b^{2}$, and uniform distributions are $\theta \sim \operatorname{Unif}(a, b)$ with $E(\theta)=(a+b) / 2$ and $\operatorname{var}(\theta)=(b-$ $a)^{2} / 12$.

\subsection{Analysis}

Our Bayesian analysis was conducted using R (version 3.2.2, R Core Team, 2015) within RStudio (version 0.99.486, RStudio Team, 2015). We constructed an MCMC chain of 10000 steps for each of the three priors. Because the Bayesian model estimates are relative and not an absolute correction 
Table 2. Increase in $H+\mathrm{LE}$ (sum of the turbulent components of the energy balance, i.e., sensible and latent heat flux) at various sites across North America after applying shadow correction to the CSAT3 time series data.

\begin{tabular}{|c|c|c|c|c|c|}
\hline \multirow[b]{2}{*}{ Site } & \multirow[b]{2}{*}{ Coordinates } & \multirow[b]{2}{*}{ Dates } & \multirow[b]{2}{*}{ Height (m) } & \multicolumn{2}{|c|}{$\begin{array}{l}\text { Percent change after } \\
\text { applying shadow correction }\end{array}$} \\
\hline & & & & Kaimal correction & $\begin{array}{r}\text { Posterior correction } \\
\text { mean } \pm \mathrm{SD}^{*}\end{array}$ \\
\hline Yuma, AZ, & $33^{\circ} 5^{\prime} \mathrm{N}$ & 6-15 June & 8.25 & $5.1 \%$ & $9.8 \pm 2.3 \%$ \\
\hline USA & $114^{\circ} 32^{\prime} \mathrm{W}$ & 2008 & & & {$[5.1,14.8 \%]$} \\
\hline Yuma, AZ, & $33^{\circ} 5^{\prime} \mathrm{N}$ & 5-14 June & 2.00 & $4.5 \%$ & $9.4 \pm 2.8 \%$ \\
\hline USA & $114^{\circ} 32^{\prime} \mathrm{W}$ & 2009 & & & {$[3.1,16.1 \%]$} \\
\hline Fraser, $\mathrm{CO}$, & $39^{\circ} 53^{\prime} 48.23^{\prime \prime} \mathrm{N}$ & 5-14 April & 27.50 & $5.6 \%$ & $9.9 \pm 1.4 \%$ \\
\hline USA & $105^{\circ} 53^{\prime} 33.87^{\prime \prime} \mathrm{W}$ & 2015 & & & {$[7.4,12.2 \%]$} \\
\hline Fraser, $\mathrm{CO}$, & $39^{\circ} 53^{\prime} 48.23^{\prime \prime} \mathrm{N}$ & 5-14 April & 6.40 & $6.8 \%$ & $11.6 \pm 1.2 \%$ \\
\hline USA & $105^{\circ} 53^{\prime} 33.87^{\prime \prime} \mathrm{W}$ & 2015 & & & {$[9.4,13.9 \%]$} \\
\hline Beltsville, MD, & $39^{\circ} 1^{\prime} 51.23^{\prime \prime} \mathrm{N}$ & 16-31 July & 4.00 & $5.5 \%$ & $10.4 \pm 2.1 \%$ \\
\hline USA & $76^{\circ} 50^{\prime} 39.40^{\prime \prime} \mathrm{W}$ & 2014 & & & {$[6.3,14.8 \%]$} \\
\hline Glacier Peak, WY, & $41^{\circ} 22^{\prime} 52^{\prime \prime} \mathrm{N}$ & 28 August- & 3.20 & $5.3 \%$ & $11.3 \pm 3.1 \%$ \\
\hline USA & $106^{\circ} 15^{\prime} 47^{\prime \prime} \mathrm{W}$ & 8 September 2015 & & & {$[4.6,19.2 \%]$} \\
\hline Agua Salud, & $9^{\circ} 13^{\prime} 31.65^{\prime \prime} \mathrm{N}$ & 6-16 November & 5.00 & $4.7 \%$ & $8.1 \pm 1.6 \%$ \\
\hline Panama & $79^{\circ} 45^{\prime} 36.41^{\prime \prime} \mathrm{W}$ & 2015 & & & {$[5.3,10.8 \%]$} \\
\hline
\end{tabular}

SD: standard deviation; * [95\% credible interval].

(see discussion in Sect. 4.1), we normalized each chain. This was done in post-processing by dividing $\alpha_{\mathrm{T}}$ by the average of $\alpha_{\mathrm{T}}$ after each time one of the 138 state variables was updated within each MCMC step. We inspected each chain using trace plots, removed the first 500 steps for burn-in, and kept 1 out of every 140 steps to eliminate autocorrelation between steps for most grid points (even after reducing to 138 state variables, a few of these were estimated from relatively fewer data, which unavoidably led to high autocorrelation between steps). This reduced each MCMC chain to 68 steps. We conducted several preliminary Bayesian analyses and used trace plots and tests for autocorrelation to determine that 10000 steps was sufficient for convergence for most of the 138 state variables defining $\alpha_{\mathrm{T}}$. Most of these parameters required little or no thinning to reduce autocorrelation between steps and could have remained as MCMC chains with 1000-10000 steps. Yet, since the goal was to create a complete 3-D correction, we decided to thin all state variables equally. Even though diagnostic tests showed that all parameters, including those with high autocorrelation, appeared to converge within 10000 steps, it is possible that these chains are still too short for proper convergence. One safeguard against this is confirming that the results from the three chains all result in similar posterior distributions (see Sect. 3.3).

Because each MCMC chain was based on a different prior, they are not replicate chains from the same Bayesian analysis. Instead, these are three separate solutions for the posterior correction. But after considering the results (see Sect. 3.3) and recognizing that, apart from normalization, the prior had minimal influence on the solution, we combined the three priors to create a single chain containing 204 independent samples of the posterior correction. We rescaled the correction to be absolute by forcing the condition that the correction will not change, on average, equatorial wind measurements (i.e., $\left(\boldsymbol{u}^{2}+\boldsymbol{v}^{2}\right)^{1 / 2}$; see discussion in Sect. 4.1). This was done by (1) applying the normalized correction to the time series of vertically mounted anemometers, (2) calculating the corrected $5 \mathrm{~min}$ standard deviations for equatorial winds, (3) performing linear regression without an intercept (i.e., model the average change in equatorial winds solely as a scaling factor) between the corrected and uncorrected standard deviations, (4) repeating this for each of the 204 posterior samples, and (5) determining the average of the 204 regression slopes. We divided all values in the normalized 3-D correction by this average scaling factor to produce our final posterior correction.

Computation of the Bayesian model was extremely intensive: completion of the three chains took upwards of 2 months of continuous computer processing (Windows 7, Intel ${ }^{\circledR}$ Core $^{\mathrm{TM}}$ i7-3630QM CPU @ 2.40 GHz processor, 1 TB solid-state hard drive, 20 GB RAM). During beta testing we attempted to estimate the 3-D correction independently for all grid points and all transducer pairs, with a single MCMC chain requiring a half year to complete. Likewise, we investigated increasing the number of grid points to obtain better resolution around the sphere as well as increasing the amount of sonic anemometer data used from the Frank et al. $(2013,2016)$ data sets. In both cases we desired an orderof-magnitude better resolution or more data, but the time required to complete a single MCMC chain quickly made these improvements impractical. Instead, we determined that 512 
grid points and $5 \%$ of the original data were optimal considering these processing constraints.

There is a slight distinction to be made between the prior corrections - which are defined as a function, $\alpha_{\mathrm{T}}(\lambda, \varphi)$, of the true longitude and latitude of the wind - and the posterior correction, which is a function, $\left.\alpha_{\mathrm{T}} \widetilde{\lambda}, \widetilde{\varphi}\right)$, where $\sim$ represents the uncorrected sonic anemometer measurement of wind location. This distinction means the posterior correction can be applied directly to the uncorrected raw data, whereas the prior should be applied iteratively (i.e., start with the uncorrected observed wind, determine the correction, update the wind measurement, determine the new wind location, update the correction, etc.). To directly compare the prior and posterior corrections, we also present our posterior correction with the wind locations recursively adjusted to approximate the true longitude and latitude. For these analyses, we smoothed the posterior spatially across the grid points with a spherical spline fit (Wahba, 1981) using R package mgcv (Wood, 2006).

We quantified the impact of shadowing on measurements of the standard deviations of winds in the three dimensions and the sensible heat flux $(H)$. This was done iteratively (i.e., for each of the 204 posterior samples) by (1) applying the posterior correction to the raw data of vertically mounted anemometers, (2) calculating the 5 min measurements, and (3) performing linear regression without an intercept between the corrected and uncorrected measurements. The 204 regression slopes were combined to form a distribution describing the relative change in each of these measurements due to shadowing. For $H$, the data were planarfit-rotated (Lee et al., 2004), time-lag-adjusted, and vaporflux-corrected (Massman and Lee, 2002) using ancillary data from the GLEES AmeriFlux site (Frank et al., 2014).

Finally, we quantified the impact of the 3-D correction on the sum of the turbulent components of the energy balance (i.e., sensible and latent (LE) heat flux) at various sites across North America (Table 2). Each site featured a CSAT3, a fast-response hygrometer, and ancillary meteorological data. Measurements of LE were calculated similar to $H$ but including the Webb-Pearman-Leuning correction (Webb et al., 1980). The impact of the 3-D correction was quantified as a distribution similar to above, except compiled from $30 \mathrm{~min}$ time periods.

\subsection{Validation experiment}

We conducted a validation experiment of the posterior 3-D correction at the Colorado State University, Agricultural Research Development and Education Center (ARDEC), Fort Collins, CO, USA $\left(40^{\circ} 39^{\prime} 7.9^{\prime \prime} \mathrm{N}, 104^{\circ} 59^{\prime} 45.7^{\prime \prime} \mathrm{W}\right)$, from 7 to 14 October 2016. Three CSAT3 sonic anemometers were mounted on an east-west boom $2 \mathrm{~m}$ above a pasture of short grass and $\sim 36 \mathrm{~m}$ south of a mature corn field. Typical winds at this site are from the north, so in this experiment we refer to cardinal $\boldsymbol{u}, \boldsymbol{v}$, and $\boldsymbol{w}$, where the measurements have been

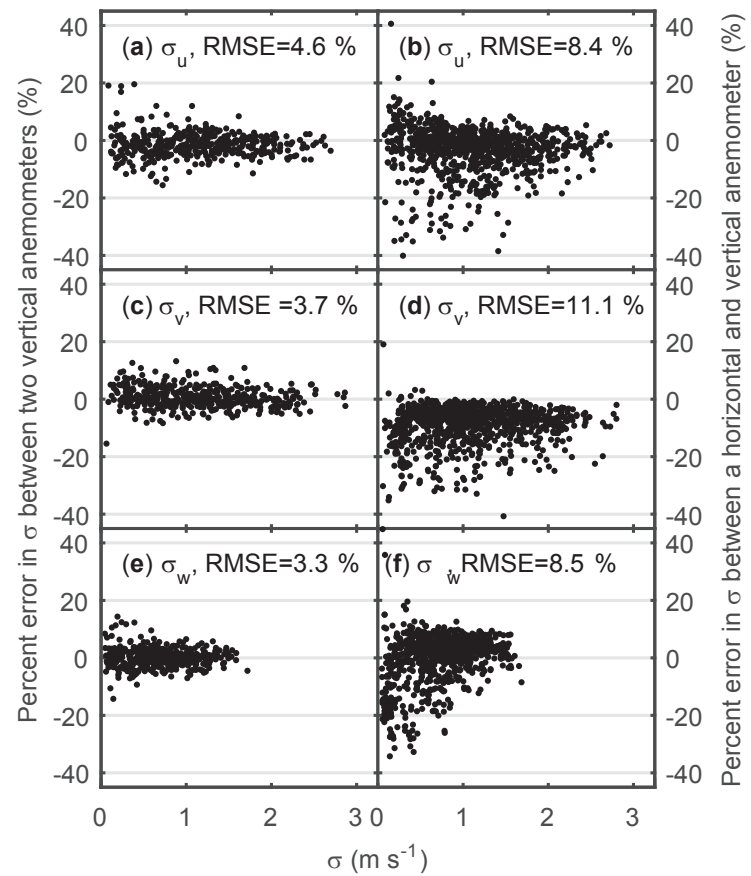

Figure 2. Uncorrected measurements of the $5 \mathrm{~min}$ standard deviation of wind $(\sigma)$ along the cardinal $(\mathbf{a}, \mathbf{b}) \boldsymbol{u},(\mathbf{c}, \mathbf{d}) \boldsymbol{v}$, and $(\mathbf{e}, \mathbf{f}) \boldsymbol{w}$ axes are not equivalent between vertically and horizontally mounted CSAT3 sonic anemometers. Data from an ideal 3-D anemometer would have similar percent errors between a horizontal and a vertical anemometer $(\mathbf{b}, \mathbf{d}, \mathbf{f})$ to those found between two anemometers mounted vertically (a, c, e). The data are from 2011 and 2013 field experiments at the GLEES AmeriFlux site (Frank et al., 2016, 2013). The 2011 data in panels (b), (d), and (f) are randomly paired between the two anemometers in different orientations. Results are summarized as root mean square error (RMSE).

rotated to north-south $(\boldsymbol{u})$, west-east $(\boldsymbol{v})$, and down-up $(\boldsymbol{w})$. One anemometer $(S / N 0869)$ was vertically mounted in the center of the boom and aimed north; a second $(S / N$ 1560) was $0.62 \mathrm{~m}$ to the east, horizontally mounted (i.e., $90^{\circ}$ rotation around its $\boldsymbol{u}$ axis), and aimed north; and a final instrument $(S / N 2385)$ was $0.58 \mathrm{~m}$ to the west and mounted askew (Fig. S1 in the Supplement). The askew mounting is unique to this validation experiment and can be defined with the unit vectors $\boldsymbol{u}$ (pointing south), $\boldsymbol{v}$ (pointing east), and $\boldsymbol{w}$ (pointing up) as $\boldsymbol{u}_{\text {askew }}=2 / 3 \boldsymbol{u}-1 / 3 \boldsymbol{v}-2 / 3 \boldsymbol{w}, \boldsymbol{v}_{\text {askew }}=2 / 3 \boldsymbol{u}+$ $2 / 3 \boldsymbol{v}+1 / 3 \boldsymbol{w}$, and $\boldsymbol{w}_{\text {askew }}=1 / 3 \boldsymbol{u}-2 / 3 \boldsymbol{v}+2 / 3 \boldsymbol{w}$. All wind velocity measurements were converted from sonic to cardinal coordinates, and all tilt angles were measured with a digital level to $0.1^{\circ}$ precision such that any mounting imperfections were taken into account. Data were measured at $20 \mathrm{~Hz}$ on a CR3000 micrologger (Campbell Scientific, Inc.). In postprocessing, both the Kaimal correction and the posterior 3-D correction were applied to the $20 \mathrm{~Hz}$ data. Data were summarized every $5 \mathrm{~min}$ as the standard deviation of wind velocity along the cardinal directions, $\sigma_{u}, \sigma_{v}$, and $\sigma_{w}$. Differences be- 


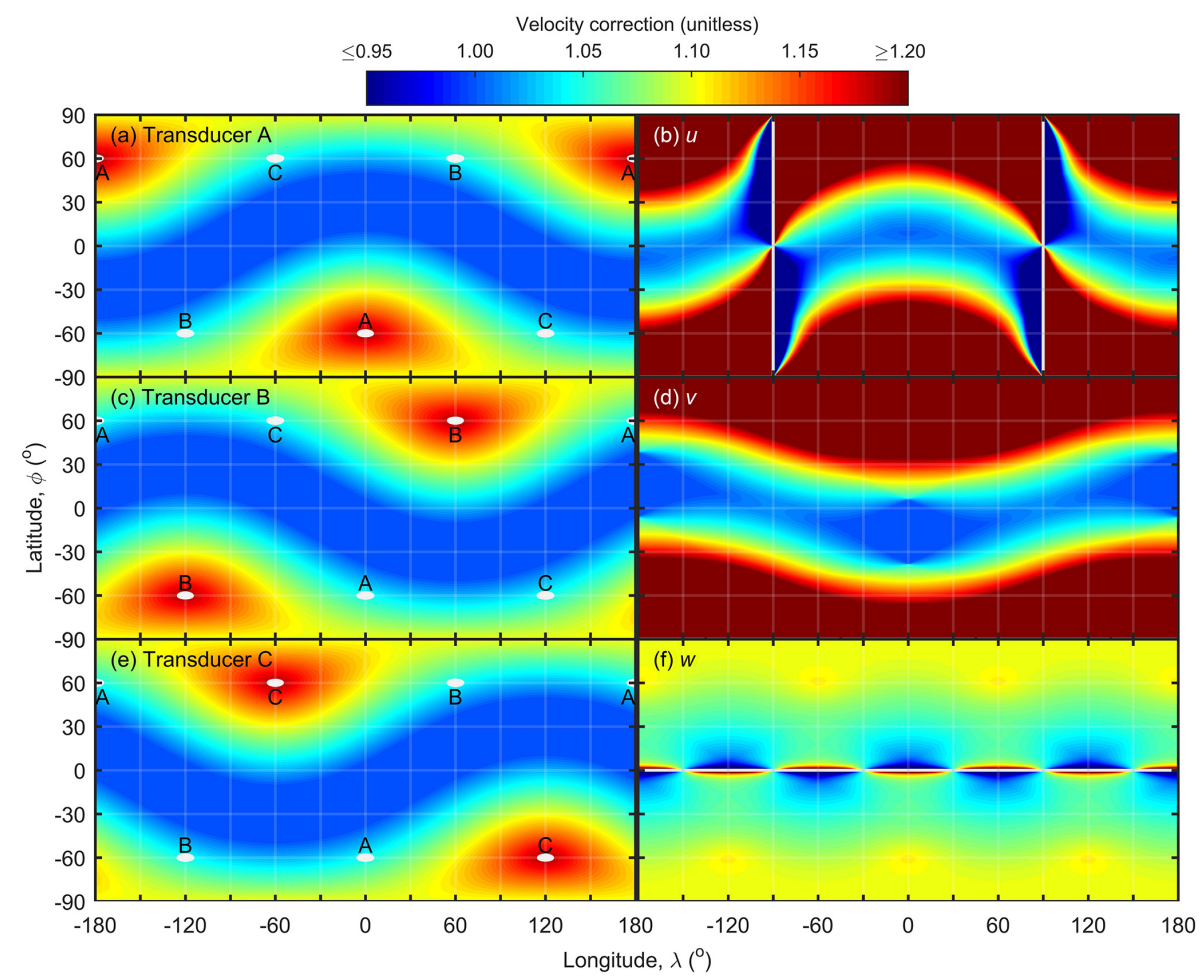

Figure 3. The Kaimal correction, one of three priors tested in this study, for the (a) A, (c) B, and (e) C transducer pairs, each represented by a white dot, of a CSAT3 sonic anemometer accounts for self-shadowing but not cross-shadowing between transducers. The same correction expressed in sonic anemometer coordinates (b) $\boldsymbol{u},(\mathbf{d}) \boldsymbol{v}$, and (f) $\boldsymbol{w}$ shows that, for near-equatorial winds, minimal correction is required for the horizontal wind components, while significant correction and instability exist in the vertical wind component $\boldsymbol{w}$. Longitude and latitude are relative to the $\boldsymbol{u}$ axis (Fig. 1).

tween anemometers are presented as root mean square of the relative error (RMSE) between measurements from the manipulated anemometers and the vertically mounted one.

\section{Results}

\subsection{No correction}

Without any shadow correction applied, measurements between a vertically and a horizontally mounted anemometer were different, which becomes clear when the variance between two vertical anemometers is taken into account (Fig. 2b, d, f vs. a, c, e). The RMSE in the 5 min standard deviation of wind along all cardinal dimensions $(\boldsymbol{u}, \boldsymbol{v}$, and $w)$ combined was $9.4 \%$ between a vertical and a horizontal anemometer, whereas the same metric between two vertical anemometers was $3.9 \%$. The largest discrepancy was along the cardinal $v$ axis, where the RMSE increased from 3.7 to $11.1 \%$ when comparing vertical and horizontal anemometers (Fig. 2c vs. d).

\subsection{The Kaimal prior correction}

The Kaimal correction is symmetrical with respect to each sonic transducer path (Fig. 3a, c, e). Yet the same correction when viewed in sonic coordinates reveals unique responses for $\boldsymbol{u}, \boldsymbol{v}$, and $\boldsymbol{w}$ (Fig. 3b, d, f). For small latitude winds, the corrections are small for $\boldsymbol{u}$ and $\boldsymbol{v}$ measurements, while those for $\boldsymbol{w}$ are higher yet unstable around the Equator (see discussion in Sect. 4.2). When the Kaimal correction was applied to the vertically mounted anemometers, there were minor increases in the $5 \mathrm{~min}$ standard deviations of $\boldsymbol{u}$ and $\boldsymbol{v}(0.8$ and $2.9 \%)$, while the increases for $\boldsymbol{w}(5.6 \%)$ and $H(5.5 \%)$ were more substantial. This correction explained some of the differences between vertically and horizontally mounted anemometers (Fig. 4), where the RMSE for all cardinal dimensions combined was $6.2 \%$, or 1.60 times greater than the same error between two vertical anemometers. The discrepancy along the cardinal $\boldsymbol{v}$ axis decreased to $6.6 \%$, or 1.86 times greater than the same error for two vertical anemometers, though some bias is still apparent (Fig. 4c vs. d). While the Kaimal correction is only one of three priors tested in our Bayesian model, it is perhaps the most accepted algorithm currently available to correct transducer shadowing in the CSAT3. 


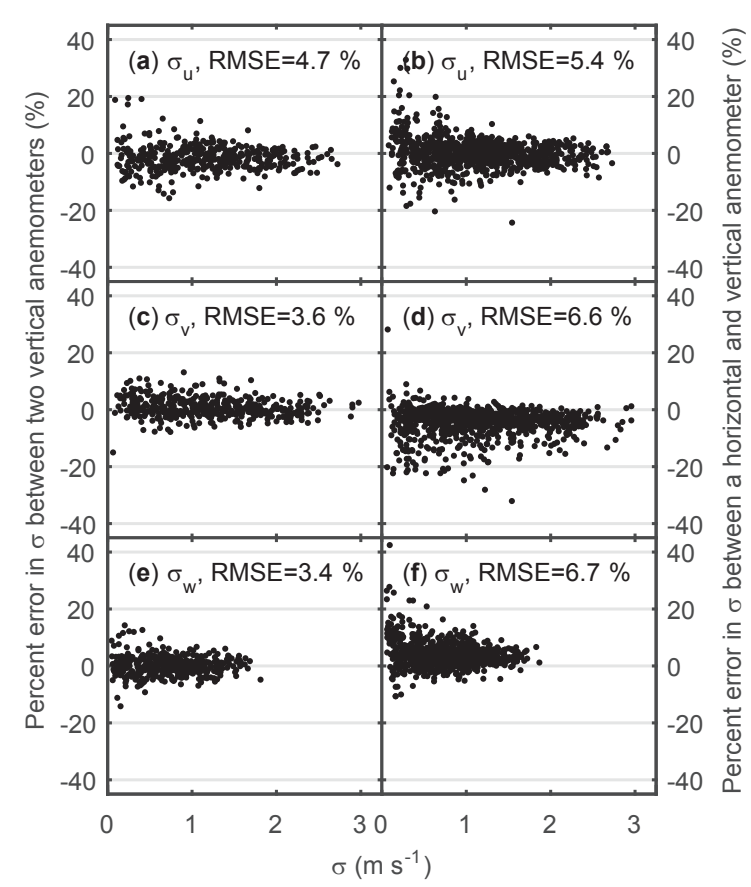

Figure 4. Kaimal-corrected measurements (i.e., one of three priors tested) of the 5 min standard deviation of wind $(\sigma)$ along the cardinal $(\mathbf{a}, \mathbf{b}) \boldsymbol{u},(\mathbf{c}, \mathbf{d}) \boldsymbol{v}$, and $(\mathbf{e}, \mathbf{f}) \boldsymbol{w}$ axes are more equivalent between vertically and horizontally mounted sonic anemometers. The percent errors between a horizontal and a vertical anemometer $(\mathbf{b}, \mathbf{d}, \mathbf{f})$ are smaller for all three cardinal dimensions than they were for the uncorrected data (Fig. 2) and are more similar to those found between two anemometers mounted vertically $(\mathbf{a}, \mathbf{c}, \mathbf{e})$. The data are from 2011 and 2013 field experiments at the GLEES AmeriFlux site (Frank et al., 2016, 2013). The 2011 data in panels (b), (d), and (f) are randomly paired between the two anemometers in different orientations. Results are summarized as RMSE.

\subsection{The Bayesian model}

Figure 5 illustrates the approach of the Bayesian model. The model initializes the 512 grid points with a prior, in this case the Kaimal correction. No matter the transducer pair or vertical versus horizontal mounting, the 3-D corrections for all cases are identical but rotated versions of a common correction based on 138 unique state variables. For a single instantaneous wind, the simultaneous corrections for all six combinations of transducer pairs and mounting orientations will be different. As the MCMC chains progress, the Bayesian model will continuously adjust each of the 138 unique state variables so that measurements from the vertically and horizontally mounted anemometers are most similar based on the univariate conditional posterior probability distribution (Eq. A13). Much of the predictive power of the model comes from resolving the inconsistencies along the cardinal $v$ axis (Fig. 2d), where vertically and horizontally mounted anemometers are likely to be most dissimilar. Specifically, a vertically mounted CSAT3 should mea- sure reasonably correct cross winds, which must flow across the entire transducer and support structure of a horizontally mounted CSAT3.

Each MCMC chain was initialized with the mean of each prior, yet after convergence their posterior corrections were remarkably similar regardless of the choice of prior correction, with one peculiarity (Fig. 6). There was a clear linear relationship between the prior correction averaged across all 512 grid points ( 1.000 for no correction, 1.040 for the Kaimal correction, and 1.080 for the double-Kaimal correction) and the magnitude of the posterior correction $(1.030,1.064$, and 1.098 , respectively) that relates to the Bayesian model estimating a relative and not absolute correction (see discussion in Sect. 4.1). The posterior correction is more than an estimate of the optimal solution, as it intrinsically accounts for the uncertainty of the correction at each of the 512 grid points (Fig. 7). Whereas each prior was defined with $10 \%$ uncertainty (Eq. 2), much of the posterior correction has much lower standard deviations, especially around the transducers where values were as low as $2.5 \%$ (Fig. 7a). These uncertainties can be expressed in sonic coordinates for the $\boldsymbol{u}, \boldsymbol{v}$, and $\boldsymbol{w}$ components, which in general show that the posterior correction is most certain for winds along each of those axes (Fig. 7b-d), with the uncertainty along the $w$ measurement ranging from 2.7 to $18.3 \%$.

Figure 8 illustrates the completion of the Bayesian model where the same posterior correction is applied to all transducer pairs and both mounting orientations. For every instantaneous wind, application of these six different corrections ultimately results in the $5 \mathrm{~min}$ standard deviations of wind along the cardinal $\boldsymbol{u}, \boldsymbol{v}$, and $\boldsymbol{w}$ axes being most similar between the two mounting orientations.

\subsection{The posterior correction}

The posterior correction for each transducer pair is presented in Fig. 9. These results take into account the recursive adjustment to the wind locations and have been smoothed with a spherical spline. Significantly more self-shadowing and cross-shadowing around the transducers are visible than for the Kaimal prior (Fig. 9a, c, e vs. Fig. 3a, c, e, in locations near all transducers). These results are more certain (i.e., low standard deviations when compared to the original $10 \%$ assigned to the prior) near the transducers, poorly constrained near the Equator (Fig. 7a), and independent of the choice of prior correction (Fig. 6). Transforming the posterior correction into sonic coordinates reveals that, similar to the Kaimal prior, minimal $\boldsymbol{u}$ and $\boldsymbol{v}$ correction is required for small latitude winds (Fig. 9b, d vs. 3b, d). But the impact of the additional transducer shadowing impacts $\boldsymbol{w}$ measurements far more than was predicted (Fig. 9f vs. Fig. 3f), where the posterior was fairly certain for latitudes greater than $\pm 13.5^{\circ}$ (Fig. 7d); the high uncertainty for near-equatorial wind is discussed in Sect. 4.2. The posterior-corrected CSAT3 was the most omnidirectional between vertically and horizontally 
(a)

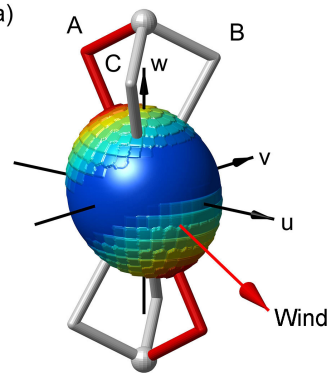

(d)

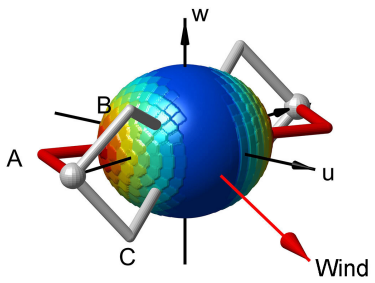

(b)

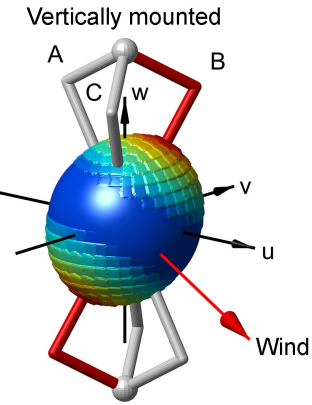

(e)

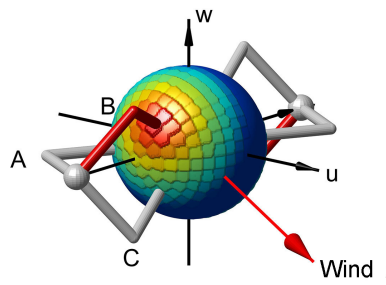

(c)

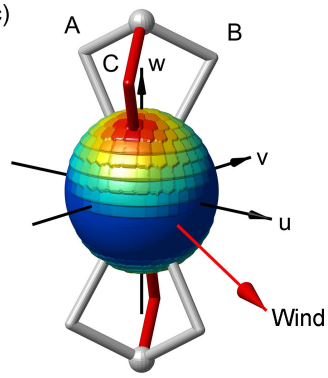

(f)

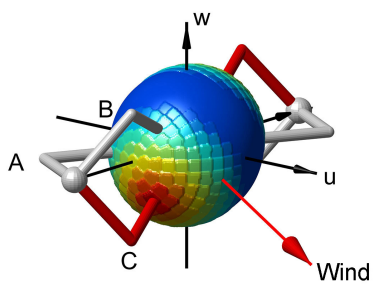

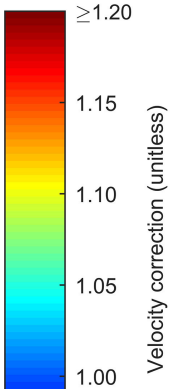

0.95

Figure 5. The Kaimal correction, one of three priors tested in this study, evaluated among 512 cells for the (a, d) A, (b, e) B, and (c, f) C transducer pairs of the CSAT3 sonic anemometer mounted either in the (a-c) typically vertical or (d-f) experimentally horizontal orientations. Though the correction is identical relative to all transducer pairs, the same instantaneous wind results in different corrections depending on the transducer pair and the orientation.

mounted anemometers (Fig. 10), where the RMSE for all cardinal dimensions combined was $5.3 \%$, or 1.36 times greater than the same error between two vertical anemometers. The discrepancy along the cardinal $v$ axis was further reduced to $4.4 \%$, which is only 1.20 times greater than the same error for two vertical anemometers, and the bias has been removed (Fig. 10d vs. 4d). When the posterior correction was applied to the vertically mounted anemometers, there were similar increases to the Kaimal correction in the 5 min standard deviations of $\boldsymbol{u}$ and $\boldsymbol{v}(0.6 \pm 0.8[-1.0,2.2] \%, 2.7 \pm 0.7$ [1.5, 4.1] $\%$, mean \pm standard deviation [ $95 \%$ credible interval], Fig. 11a-b). But compared to the Kaimal correction, the increases in $\boldsymbol{w}(10.6 \pm 1.7[7.6,13.9] \%)$ and $H(9.9 \pm 1.6[7.2$, 12.6] \%) were substantial and significantly higher (Fig. 11cd). We provide the MCMC chain for the final posterior correction in the Supplement as a tool for researchers to evaluate in other sonic anemometer studies, to examine the uncertainty in ecosystem flux measurements, and to investigate surface energy balance closure.

\subsection{Turbulent components of the ecosystem energy balance across a continent}

We applied the posterior correction to various sites across North America that deploy the CSAT3 in their eddycovariance instrumentation (Table 2). The estimated increase in $H+\mathrm{LE}$ at these sites ranged from 8.1 to $11.6 \%$ with an average standard deviation and $95 \%$ credible interval of $\pm 1.9 \%$ and $6.1-13.8 \%$, respectively. For all but one site, the increase in $H+$ LE was significantly higher than the increase due to the Kaimal correction. At the $2 \mathrm{~m}$ Yuma, AZ site, the lack of significance is related to anomalously low instantaneous wind latitudes for which the $\boldsymbol{w}$ correction is most uncertain (Fig. 7d).

\subsection{Validation of the posterior correction}

The validation experiment was conducted during excellent fall weather with no precipitation, where winds averaged $2.0 \pm 1.2 \mathrm{~m} \mathrm{~s}^{-1}$, maximum sustained gusts were $7.8 \mathrm{~m} \mathrm{~s}^{-1}$, $38 \%$ of the winds were from the northeast $\left(45^{\circ}\right)$ to northnorthwest $\left(337.5^{\circ}\right), 25 \%$ of the winds were from the southeast $\left(135^{\circ}\right)$ to south $\left(180^{\circ}\right)$, and during the other times there were some occasional westerly winds. Results are summarized in Table 3. The RMSE differences between a horizontally mounted anemometer and a vertically mounted anemometer were large (12.6-16.5\%) for uncorrected measurements. Applying the Kaimal correction to these anemometers reduced the RMSE differences in $\sigma_{u}$ and $\sigma_{v}(8.5$ and $11.4 \%)$ but increased the difference in $\sigma_{w}$ $(17.5 \%)$. Compared to the uncorrected data, the average posterior correction decreased the RMSE differences in all directions, though only the reduction in $\sigma_{v}(8.0-12.2 \%)$ was statistically lower (i.e., $95 \%$ credible interval). Compared to the Kaimal correction, the average posterior correction was larger for $\sigma_{u}$ but lower for $\sigma_{v}$ and $\sigma_{w}$, with the reduction in $\sigma_{w}(11.8-15.9 \%)$ being statistically lower than with the Kaimal-corrected data. The RMSE differences between 

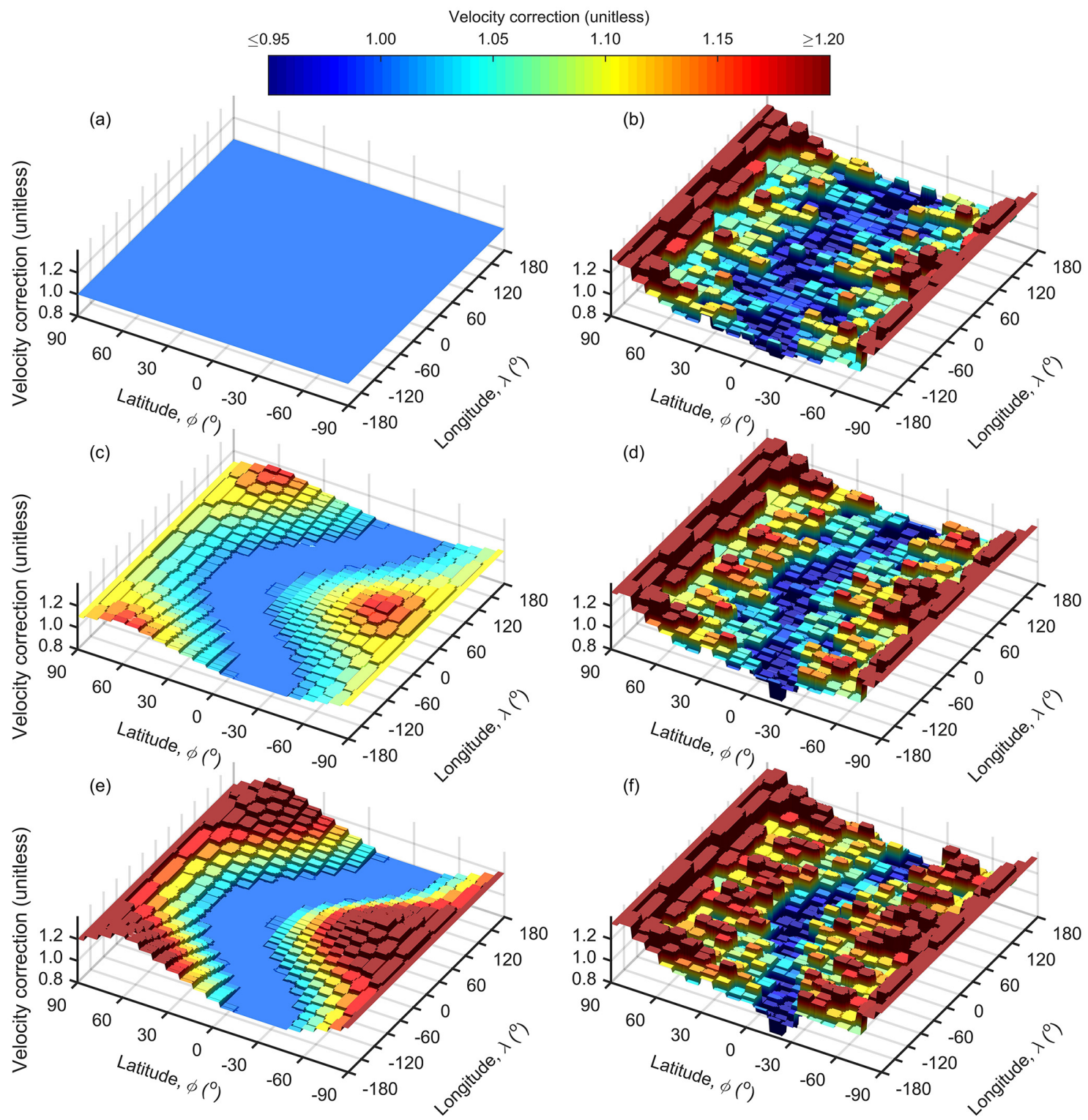

Figure 6. The A transducer pair correction evaluated among 512 cells for the three prior corrections tested in this study: (a) flat, (c) Kaimal, and (e) double Kaimal, with their corresponding unnormalized posterior corrections (b), (d), and (f), respectively. All posteriors have similar relative topography. They differ in absolute scaling, where priors with higher absolute magnitude result in posteriors with higher absolute magnitude, which is apparent from the different colorings.

an askew-mounted anemometer and a vertically mounted anemometer were small/moderate for $\sigma_{u}$ and $\sigma_{v}$ (6.7 and $11.3 \%)$ and large for $\sigma_{w}(14.7 \%)$ for uncorrected measurements. Applying the Kaimal correction to these anemometers reduced the RMSE differences in all directions (4.4-13.5\%). The standard deviations for the RMSE differences using the posterior correction were higher for the askew manipulation $(1.5-2.4 \%)$ than they were for the horizontal manipulation $(1.1-1.3 \%)$. Compared to the uncorrected data, the average posterior correction increased the RMSE difference for $\sigma_{u}$ $(8.6 \%)$ but decreased the differences for $\sigma_{v}$ and $\sigma_{w}(10.3$ and $13.9 \%$ ), though none of these changes were statistically significant. Compared to the Kaimal correction, the average posterior correction increased the RMSE differences for all directions, with the differences in $\sigma_{u}(6.2-11.6 \%)$ and $\sigma_{v}$ $(7.2-13.5 \%)$ being statistically larger. 


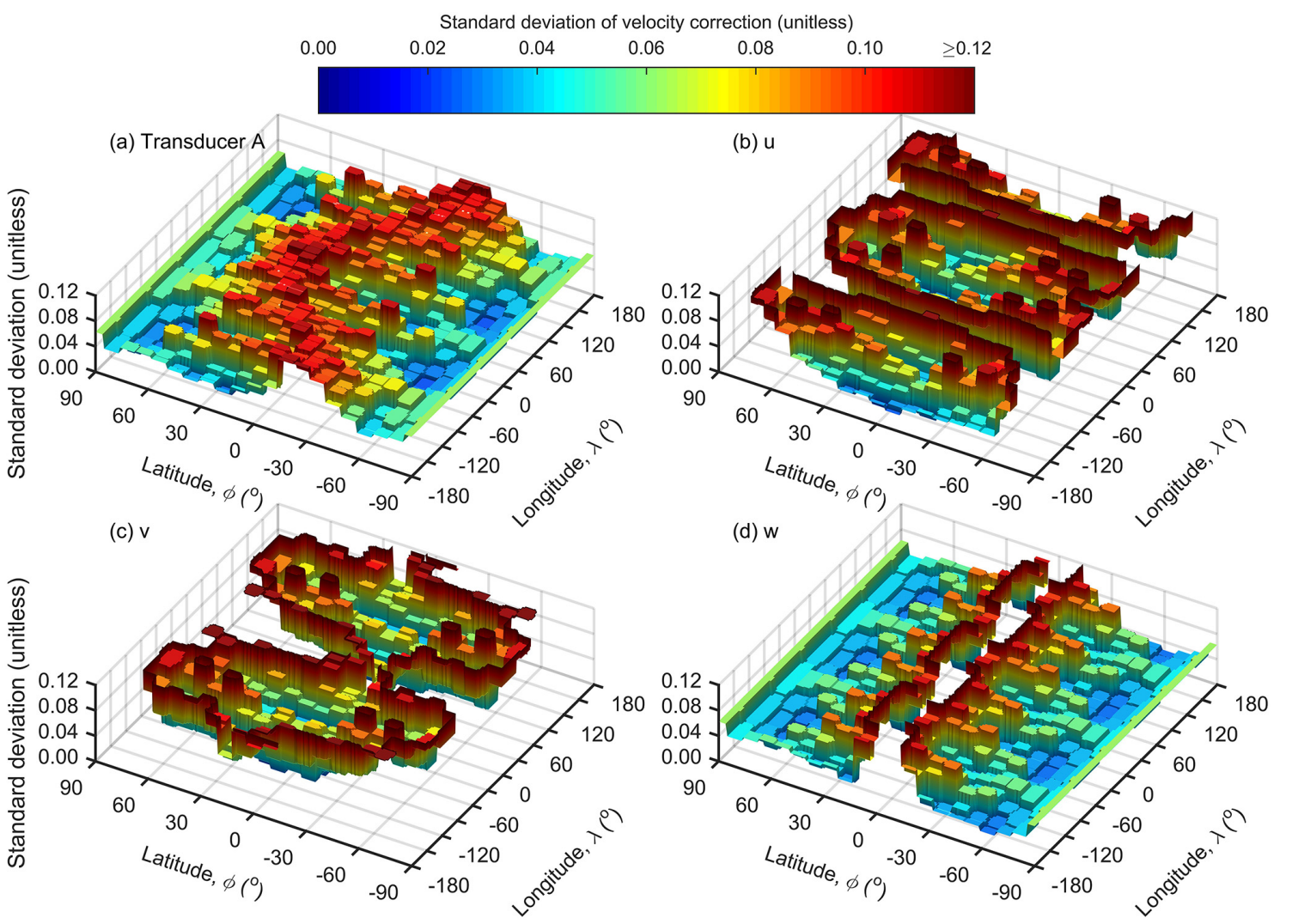

Figure 7. Standard deviations of the posterior correction for (a) the A transducer pair and the wind velocities (b) $\boldsymbol{u}$, (c) $\boldsymbol{v}$, and (d) $\boldsymbol{w}$. When compared to the standard deviation of the prior which was defined as 0.1 , the transducer correction is more certain in regions with higher topography (Fig. 6). The results in CSAT3 sonic coordinates reflect both the uncertainty in the transducer correction plus cancelation and amplification of errors due to the coordinate transformation. The posterior correction for $\boldsymbol{u}, \boldsymbol{v}$, and $\boldsymbol{w}$ is most certain for winds along the $\boldsymbol{u}$, $\boldsymbol{v}$, and $\boldsymbol{w}$ axes, respectively.

Table 3. Results of a validation experiment of CSAT3 sonic anemometers at ARDEC, CO, showing the relative error in 5 min standard deviation of wind $(\sigma)$ along the cardinal $\boldsymbol{u}, \boldsymbol{v}$, and $\boldsymbol{w}$ axes between a vertical instrument and one mounted horizontal and one mounted askew. All anemometers were compared uncorrected, with the Kaimal correction, and with the posterior correction.

\begin{tabular}{|c|c|c|c|c|}
\hline \multirow[b]{2}{*}{ Manipulation } & \multirow[b]{2}{*}{$\begin{array}{c}\text { Cardinal } \\
\text { measurement }\end{array}$} & \multicolumn{3}{|c|}{$\begin{array}{l}\text { RMSE in } \sigma \text { between a } \\
\text { manipulated and vertical anemometer }(\%)\end{array}$} \\
\hline & & Uncorrected & $\begin{array}{r}\text { Kaimal } \\
\text { correction }\end{array}$ & $\begin{array}{r}\text { Posterior correction } \\
\text { mean } \pm \mathrm{SD}^{*}\end{array}$ \\
\hline \multirow[t]{3}{*}{ Horizontal } & $\sigma_{u}$ & $12.6 \%$ & $8.5 \%$ & $\begin{array}{l}10.5 \pm 1.3 \% \\
{[8.4,13.4 \%]}\end{array}$ \\
\hline & $\sigma_{v}$ & $16.5 \%$ & $11.4 \%$ & $\begin{array}{r}9.8 \pm 1.1 \% \\
{[8.0,12.2 \%]}\end{array}$ \\
\hline & $\sigma_{w}$ & $15.0 \%$ & $17.5 \%$ & $\begin{array}{r}13.4 \pm 1.1 \% \\
{[11.8,15.9 \%]}\end{array}$ \\
\hline \multirow[t]{3}{*}{ Askew } & $\sigma_{u}$ & $6.7 \%$ & $4.4 \%$ & $\begin{array}{r}8.6 \pm 1.5 \% \\
{[6.2,11.6 \%]}\end{array}$ \\
\hline & $\sigma_{v}$ & $11.3 \%$ & $6.0 \%$ & $\begin{array}{l}10.3 \pm 1.7 \% \\
{[7.2,13.5 \%]}\end{array}$ \\
\hline & $\sigma_{w}$ & $14.7 \%$ & $13.5 \%$ & $\begin{array}{l}13.9 \pm 2.4 \% \\
{[9.9,19.4 \%]}\end{array}$ \\
\hline
\end{tabular}

RMSE: root mean square error; SD: standard deviation; ${ }^{*}$ [95\% credible interval]. 
(a)

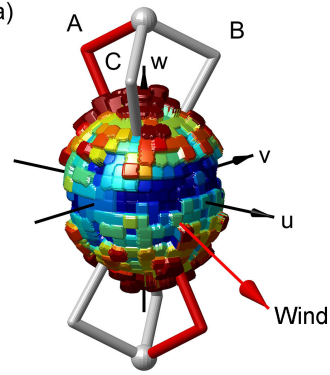

(d)

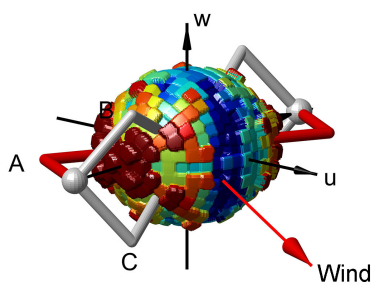

(b)

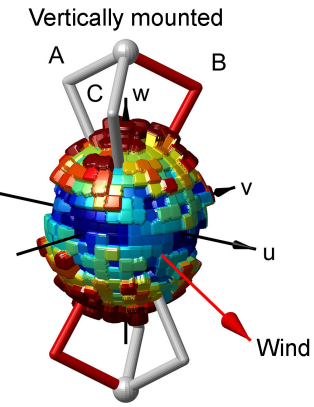

(e)

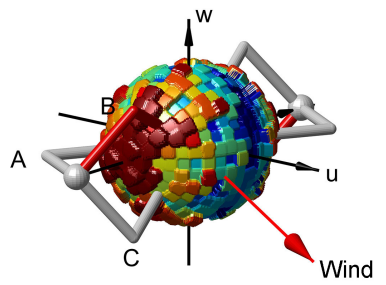

(c)

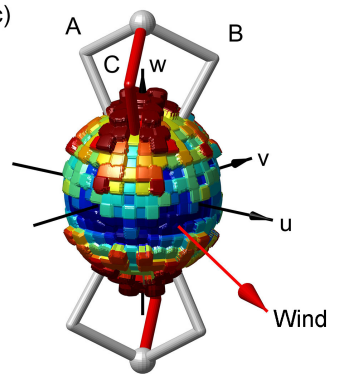

(f)

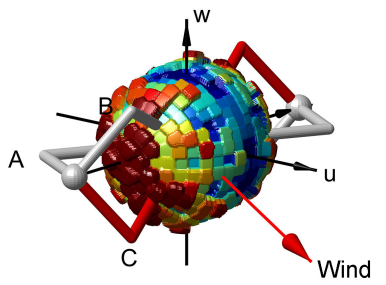

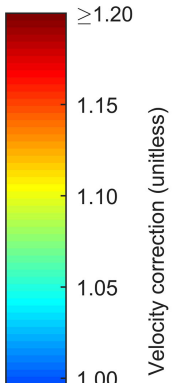

0.95

Figure 8. The posterior correction evaluated for the (a, d) A, (b, e) B, and (c, f) C transducer pairs of the CSAT3 sonic anemometer mounted either in the (a-c) typically vertical or $(\mathbf{d}-\mathbf{f})$ experimentally horizontal orientations. The correction is identical relative to all transducer pairs and is constructed from 512 cells with 138 unique values. The Bayesian model adjusts these values to simultaneously correct the same instantaneous wind measured from different transducer pairs and orientations in order to produce similar cardinal $\boldsymbol{u}, \boldsymbol{v}$, and $\boldsymbol{w}$ wind statistics (Fig. 10).

\section{Discussion}

\subsection{An omnidirectional standard}

Perhaps the most important shortcoming in almost every sonic anemometer study is the lack of a standard wind measurement to compare against. A fundamental problem is that the principle of sonic measurements (Barrett and Suomi, 1949; Kaimal and Businger, 1963) involves the observer effect; i.e., it is virtually impossible for sonic transducers to observe air parcels without influencing them (Buks et al., 1998). Thus, any method that relies on a sonic anemometer measurement as an absolute standard is flawed to an extent. And while we are justified to believe that some sonic anemometer measurements are more accurate that others (Frank et al., 2016), it is tenuous to choose any sonic anemometer measurement as a standard. Then, what are the alternatives? Wind tunnels are extremely useful (Horst et al., 2015; van der Molen et al., 2004), yet it is debatable that such laminar or quasi-laminar calibrations are transferrable to turbulent field conditions (Hogstrom and Smedman, 2004). And, while other new technologies such as Doppler Lidar exist (Sathe et al., 2011; Dellwik et al., 2015), their application as a field reference standard has been limited.

What we address is the general problem of determining a calibration given an unknown standard or nothing to compare against. Whether this problem exists in medicine (Lu et al., 1997), acoustics (MacLean, 1940; Monnier et al., 2012), or micrometeorology with respect to calibrating sonic anemometry in turbulent flow fields, all approaches have a commonality of testing the relative consistency of a response to unknown signals. In our situation, we hold the 3-D sonic anemometer to an omnidirectional standard of relative consistency and contend that the correction that best achieves this standard is statistically the most likely 3-D calibration. A CSAT3 without any 3-D shadow correction is clearly not omnidirectional (Fig. 2) as measurements depend on the instrument's orientation. A CSAT3 with the Kaimal transducer shadow correction is better at meeting this standard (Fig. 4). However, the posterior 3-D correction is remarkably effective in making the CSAT3 omnidirectional (Fig. 10). Because the posterior correction closely achieves the omnidirectional standard, we support our first hypothesis and argue that it is the most accurate correction, in general, for the three dimensions of the CSAT3. Whether or not the posterior correction reveals meaningful information regarding vertical winds and turbulent fluxes is another matter discussed below.

A consequence of the omnidirectional standard is that implicitly this produces only relative results. Indeed, our Bayesian posterior has no meaning in an absolute sense without the additional constraint that equatorial winds should be unchanged by the correction. We do not specify the 3-D correction at any of the grid points, nor we do we specify a reference or true condition for the standard deviation of wind during any $5 \mathrm{~min}$ period. Because of this, the parameter es- 


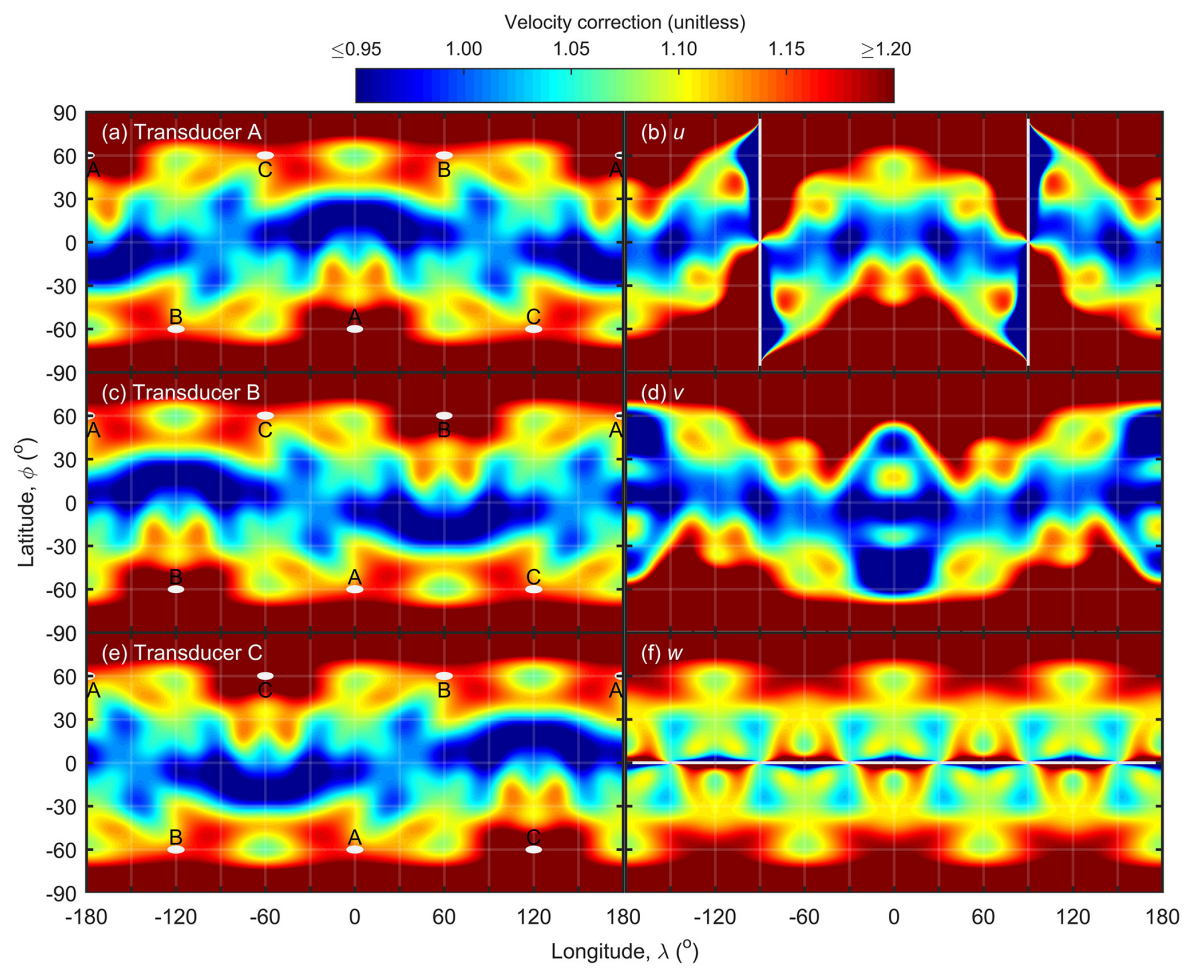

Figure 9. The posterior correction for the (a) A, (c) B, and (e) C transducer pairs, each represented by a white dot, of a CSAT3 sonic anemometer accounts for both self-shadowing and cross-shadowing between transducers. The same correction expressed in sonic anemometer coordinates (b) $\boldsymbol{u},(\mathbf{d}) \boldsymbol{v}$, and (f) $\boldsymbol{w}$ shows that, for near-equatorial winds, minimal correction is required for the horizontal wind components, while even more correction exists in the vertical wind component $\boldsymbol{w}$ than was present with the Kaimal correction (Fig. 3f). Longitude and latitude are relative to the $\boldsymbol{u}$ axis (Fig. 1).

timates for $\widetilde{\sigma}_{C}$ and $\alpha_{T}$ only have meaning relative to each other. This issue is confounded by the choice of prior distributions, which vary dramatically in shape but produce similar posteriors except for differences in their absolute magnitudes (Fig. 6); i.e., higher magnitude priors produce higher magnitude posteriors. Which absolute magnitude is correct? Without specifying an absolute standard, the answer is none of them. To facilitate comparison and combination of the posteriors, we normalized the three MCMC chains.

There is a clear need to specify an absolute standard to reference our results. Without one, our normalized posterior correction reduced the $5 \mathrm{~min}$ standard deviations for equatorial winds (i.e., the $\boldsymbol{u}-\boldsymbol{v}$ plane) by $7 \%$. Does this make physical sense? No. The idea that equatorial winds should not be changed is consistent with the expectation that the CSAT3 accurately measures equatorial winds, something that has been demonstrated in both wind tunnels and field campaigns (Yahaya and Frangi, 2004; Friebel et al., 2009). Even the Kaimal correction, which is an absolute correction, predicts $<0.1 \%$ error in our measurements of equatorial winds. Because the omnidirectional standard is only relative, we impose an additional absolute standard by defining the average correction for equatorial winds to be 0 , which is simply achieved by scaling the normalized posterior correction by
$7 \%$. While there certainly is some leeway in this constraint, if the normalized posterior correction were scaled by anything other than $7 \pm 1.4 \%$ then the correction to horizontal winds would be significantly different $(95 \%$ credible interval) than both 0 and the Kaimal correction (Fig. 11a-b) and would run counter to our belief that the CSAT3 makes reasonably accurate measurements of horizontal winds.

\subsection{Impact on vertical wind measurements and sensible heat flux}

Recent studies have questioned the accuracy of CSAT3 vertical wind velocity measurements (Frank et al., 2013; Kochendorfer et al., 2012), culminating with Horst et al. (2015) and Frank et al. (2016), who identified the anemometer's lack of transducer shadowing correction as the root cause. Quantifying the inaccuracy and determining how to fix this problem have been a challenge. While each of these studies estimated different errors in $w$ at their field sites $(3.5 \%$, Horst et al., 2015; 6-10\%, Frank et al., 2013; 5.5-12.5\%, Frank et al., 2016; and 14\%, Kochendorfer et al., 2012), it was not until Horst et al. (2015) proposed the application of the Kaimal correction (Kaimal, 1979) that a mechanistic explanation was used to quantify the underestimate. Whether or 


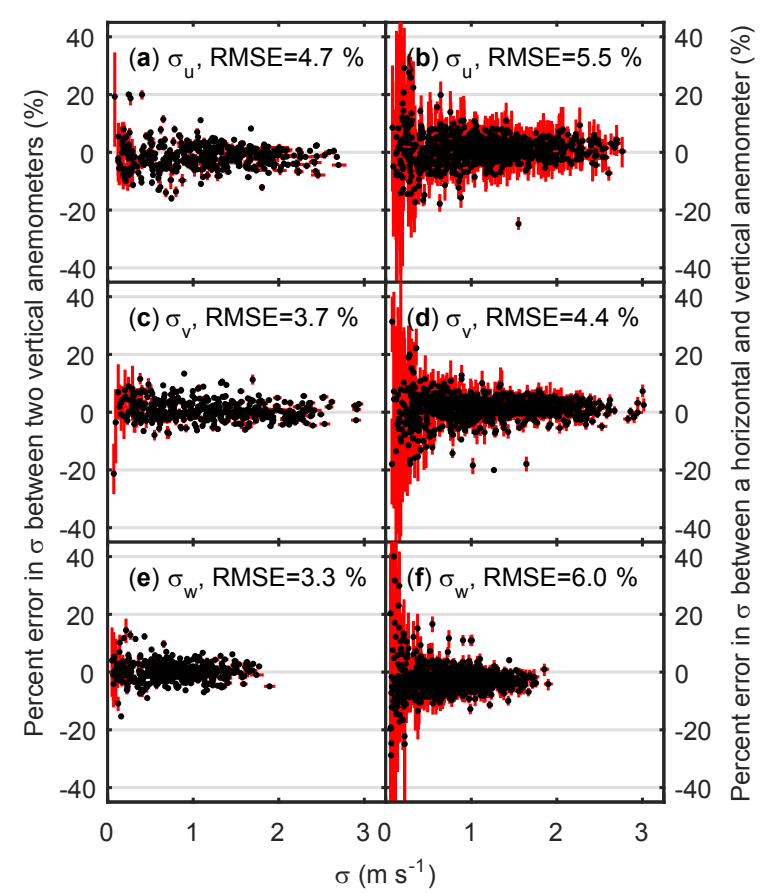

Figure 10. Posterior-corrected measurements of the 5 min standard deviation of wind $(\sigma)$ along the cardinal $(\mathbf{a}, \mathbf{b}) \boldsymbol{u}$, (c, d) $\boldsymbol{v}$, and $(\mathbf{e}, \mathbf{f}) \boldsymbol{w}$ axes are most equivalent between vertically and horizontally mounted sonic anemometers than with either the uncorrected (Fig. 2) or Kaimal-corrected data (Fig. 4). The percent errors between a horizontal and a vertical anemometer are small $(\mathbf{b}, \mathbf{d}, \mathbf{f})$, especially for the cardinal $\boldsymbol{v}$ dimension (d), and are similar to those found between two anemometers mounted vertically (a, c, e). The data are from 2011 and 2013 field experiments at the GLEES AmeriFlux site (Frank et al., 2016, 2013). The 2011 data in panels (b), (d), and (f) are randomly paired between the two anemometers in different orientations. Results are summarized as RMSE. The red lines are $95 \%$ credible intervals.

not the Kaimal correction is sufficient is a matter of debate, but it currently represents the best prior knowledge to explain the CSAT3's shortcomings.

Solely the fact that the posterior correction makes the CSAT3 more omnidirectional does not imply that field measurements of vertical wind and turbulent fluxes are impacted, nor does this assure that these impacts would be due to anything more than chance. Even with the uncertainty in the posterior $\boldsymbol{w}$ correction explicitly quantified (Fig. 7d), it is difficult to foresee if $\boldsymbol{w}$ is significantly impacted without applying the posterior correction to actual data. A powerful attribute of the Bayesian analysis is that the posterior correction can be applied to raw data to produce probability distribution estimates for $\boldsymbol{w}$ and $H$ from which statistical inferences can be made. Using GLEES data, Fig. 11c-d confirms that to achieve an omnidirectional sensor (Fig. 10) with minimal change to horizontal winds (Fig. 11a-b) the required correction will increase both $\boldsymbol{w}$ and $H$ by an average of 10.6 and $9.9 \%$, which is significantly more (>95\% credible interval)

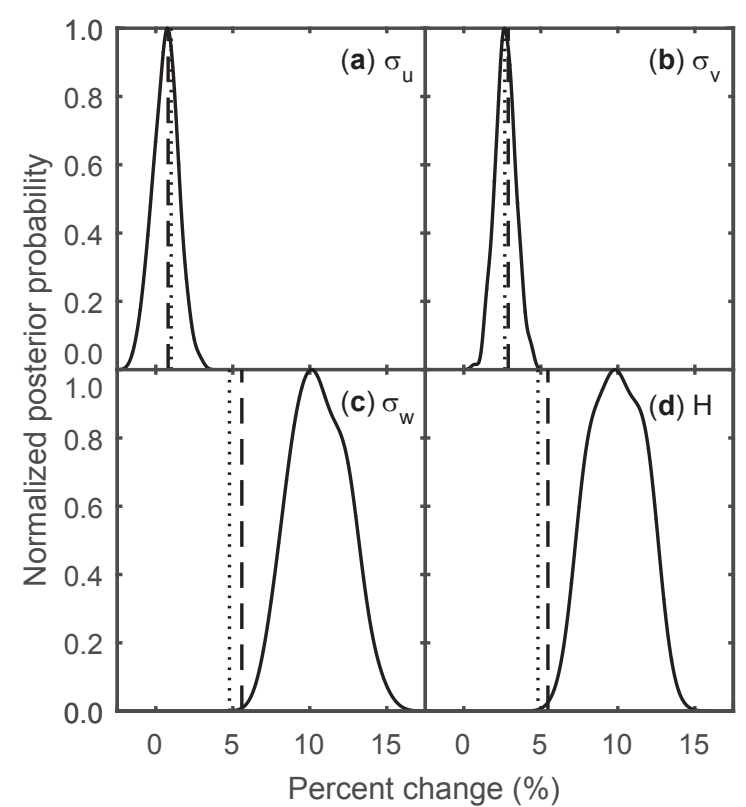

Figure 11. Though application of the Kaimal (dashed lines) and posterior (solid lines) corrections results in similar changes to the 5 min standard deviations of wind ( $\sigma$ ) along the (a) $\boldsymbol{u}$ and (b) $\boldsymbol{v}$ axes, application of the posterior correction results in significantly higher (95\% credible interval) (c) winds along the $w$ axis and (d) sensible heat flux $(H)$. The dotted lines are an alternate formulation of the Kaimal correction proposed by Wyngaard and Zhang (1985) and used in Horst et al. (2015). Data are for vertically mounted anemometers only.

than predicted by the Kaimal prior. We argue that this significant increase in the vertical wind occurs because the posterior correction more accurately accounts for all shadowing between transducers (Fig. 9 vs. 3); therefore we support our second hypothesis.

Also of note, there are instabilities in the prior and posterior $\boldsymbol{w}$ corrections for near-equatorial winds that occur at latitudes less than $\pm 4^{\circ}$ (six inflection points around the Equator, Figs. $3 \mathrm{f}$ and $9 \mathrm{f})$. The mathematical cause for these instabilities and the locations of the inflection points are derived in Appendix A2, and unless the corrections for the three transducers are exactly equal everywhere around the Equator these instabilities will exist. The existence of these instabilities should cause concern for eddy-covariance measurements. The ultimate impact of this phenomenon is difficult to know, because, on one hand, $\boldsymbol{w}$ for latitudes less than $\pm 4^{\circ}$ are by definition very small, but, on the other, these eddies constitute a large proportion of winds that exist under field conditions, and their correction is currently unpredictable. For example, at GLEES $30 \%$ of winds occur at latitudes within $\pm 4^{\circ}$ (unpublished analysis of Fig. 4 from Frank et al., 2016). It is unknown how aggressively the correction for these winds approaches $\pm \infty$ or if more inflection points actually occur. For all non-orthogonal geometries, not just the 
CSAT3, if any transducer shadowing occurs at the Equator, there will be instabilities in the $\boldsymbol{w}$ correction.

\subsection{Impact across global flux networks}

Energy balance is a fundamental ecosystem concept where the flow of available energy into an ecosystem influences the microclimate, drives photosynthesis, and establishes trophic levels among the biota (Odum, 1957; Fisher and Likens, 1973; Teal, 1962). Yet eddy-covariance studies of ecosystem fluxes seldom delve into details of energy flow beyond the generation of sensible and latent heat. It is often stated that most eddy-covariance sites underestimate these turbulent components of the energy balance by 10-20\% when compared to the available energy (Wilson et al., 2002; Foken, 2008; Stoy et al., 2013; Leuning et al., 2012; Franssen et al., 2010). Even when sites thoroughly account for lesser components such as energy stored in the biomass or canopy air, the turbulent energy can still be $1-14 \%$ underestimated (Heilman et al., 2009; Oliphant et al., 2004; Barr et al., 2006; Wang et al., 2012). It is common for sites to deal with this problem by forcing energy balance closure by increasing $H$ and/or LE (Heilman et al., 2009; Oliphant et al., 2004; Twine et al., 2000; Scott et al., 2004) or even carbon fluxes (Barr et al., 2006) by the percent of the energy imbalance. Is there a mechanistic reason why so many sites believe their turbulent fluxes are underestimated? While it is difficult to generalize for every site, one similarity among these studies (Heilman et al., 2009; Oliphant et al., 2004; Barr et al., 2006; Wang et al., 2012; Twine et al., 2000; Scott et al., 2004) is they all feature a CSAT3, as do $\sim 60 \%$ of all sites in the AmeriFlux network (unpublished summary of 150 of the 228 sites where anemometer information was available, list accessed at http://ameriflux.lbl.gov/ in November 2015) and numerous sites distributed across the world within FLUXNET (http://fluxnet.fluxdata.org/).

After applying the posterior correction to the CSAT3 at our site, measurements of one of the energy balance components, $H$, increased $9.9 \pm 1.6 \%$, which is about twice the $5.5 \%$ increase predicted by the Kaimal correction (Fig. 11) (note that the field experiments were conducted without a colocated fast-response hygrometer; hence we do not estimate the impact on LE at our site). However, we must consider that our field site in Wyoming is unusual, with extreme wind and turbulence, and where summer friction velocity $\left(\boldsymbol{u}_{*}\right)$ averages $0.6 \mathrm{~m} \mathrm{~s}^{-1}$ (Frank et al., 2016). While this made GLEES a good location to conduct the turbulent field experiments that led to the development of the posterior correction, do our results lead to similar impacts on ecosystem fluxes elsewhere? To answer this, we applied the posterior correction to eddy-covariance measurements at various sites across North America that employ the CSAT3 (Table 2). We found that the sum of the turbulent components of the energy balance (sensible plus latent heat flux) increased on average between 8 and $12 \%$, with the average $95 \%$ credible interval being
6-14\%. At most sites this was significantly higher than applying the Kaimal correction. Thus, it is highly probable that at flux sites that employ the CSAT3 sonic anemometer the posterior correction will significantly increase the turbulent components of the energy budget and explain much of the ubiquitous energy imbalance problem; therefore we support our third hypothesis.

Are the results from this study applicable to the nonorthogonal sonic anemometers produced by other manufacturers? Possibly. Frank et al. (2016) showed that the Applied Technologies, Inc. A-probe shares a similar transducer geometry, a lack of a shadow correction algorithm, and similar differences between vertically and horizontally mounted anemometers, so it would be reasonable to expect a similar 3-D correction for that instrument. But other manufacturers do apply wake corrections in their firmware that are traceable to wind tunnel calibrations. Are these adequate? Maybe not, as non-orthogonal anemometers from other manufacturers have been implicated to erroneously measure the vertical wind (Kochendorfer et al., 2012; Nakai et al., 2014; Nakai and Shimoyama, 2012). Without details of the calibrations or the wake corrections it is difficult to know. Regardless, for any non-orthogonal sonic anemometer with vertically oriented transducers, equatorial instabilities are likely to exist (Appendix A2) that would be extremely difficult to characterize with only a series of wind tunnel calibrations. One benefit of our methodology is that it allows an independent check on the sufficiency of these wake corrections. If such an instrument failed to consistently measure three-dimensional winds (i.e., it responds like Fig. 2), then our methodology would estimate a posterior correction that could correct a wake-corrected anemometer. Because $~ 90 \%$ of all AmeriFlux sites use non-orthogonal sonic anemometers (Frank et al., 2013; Nakai et al., 2014), it would be appropriate to investigate this issue for all non-orthogonal sonic anemometer designs.

\subsection{The next step}

While these results reveal much about the nature of shadowing in a non-orthogonal sonic anemometer, there is much more to be done. First, due to the intense computational burden of this analysis we never fully utilized our data. While we only analyzed $5 \%$ of the available data, limited the $3-\mathrm{D}$ correction to approximately $\pm 5^{\circ}$ resolution and only 138 unique corrections, and terminated the Bayesian MCMC chains after only 10000 steps, it still took months of continuous processing with extensive memory usage to produce these results. Obviously there is an opportunity to adapt this analysis to run on multiple cores or a supercomputer. As we developed our analysis, it became apparent that with more data the standard deviations of the posterior distribution improved; we foresee that with 20 times more data the uncertainty in the posterior correction would be further reduced. Adaptation to a high-performance computer will allow for a more precise 
grid, longer MCMC chains, and a lower standard deviation of the posterior distribution.

Our results draw extensively on the symmetry of the CSAT3, which fails to account for the upper and lower mounting arms that extend back into the electronics housing and support block. We beta-tested our model to solve for the 3-D correction independently for each transducer and for all grid points around the sphere. We abandoned this because winds at GLEES are fairly unidirectional, causing many of the grid points to be poorly characterized. Plus with an orderof-magnitude more unique grid points to solve, the computation took over 5 months to complete just one MCMC chain! There is a middle ground between assuming symmetry and pooling data; i.e., the correction for the A transducer pair could be considered symmetrical along the $\boldsymbol{u}-\boldsymbol{w}$ plane, and the corrections for transducer pairs B and C are mirror images of each other. In addition to solving the problem with fewer assumptions of symmetry, more experimental manipulations should be tested. We only tested a $90^{\circ}$ rotation along the $\boldsymbol{u}$ axis, but there are limitless other manipulations that would help characterize the shadowing around the entire 3-D space surrounding an anemometer. Our model could easily be adapted to handle different manipulations using Eq. (7). This equation can be expanded to account for a limitless number of manipulations within the same analysis.

Sonic anemometer corrections should be verified and validated. There is an opportunity to statistically cross-validate the posterior 3-D correction with subsets of the other $95 \%$ of available data; we decided against this because the $5 \%$ used was already partitioned equally throughout the full data set; plus, analyzing multiple rounds of training and validation data sets would take additional months of computation. Instead of a statistical cross-validation analysis, we conducted a validation field experiment to determine (1) if our results are reproducible and (2) if they can explain other manipulations. From this, we first conclude that our results are reproducible. In both our main experiments at GLEES and the validation experiment at ARDEC, there was improved agreement between vertically and horizontally mounted anemometers when using the posterior correction versus the Kaimal correction or no correction (Table 3). The largest differences between anemometers was for $\sigma_{v}$ (11.1 and $16.5 \%$, Fig. 2d, Table 3), which were reduced with the Kaimal correction (6.6 and $11.4 \%$, Fig. 4d, Table 3 ) and then further improved with the posterior correction (4.4 and 9.8\%, Fig. 10d, Table 3). In both analyses, the differences in $\sigma_{u}$ were reduced with either correction, but the best performance was the Kaimal prior (Fig. 4b vs. 10b, Table 3). Finally, in both cases the differences in $\sigma_{w}$ were smallest using the posterior correction (Fig. 4f vs. 10f, Table 3). Moreover, we justify our validation because it involved an independent data set that was collected at a different field site, over radically different terrain and vegetation, and using anemometers with different serial numbers. We are less confident that our posterior correction can explain all manipulations. The differences in $\sigma_{u}$ and $\sigma_{v}$ between vertically and askew-mounted anemometers were significantly better with the Kaimal correction (Table 3). It is important to note, however, that these differences were the smallest of all the comparisons ("Uncorrected" column in Table 3); i.e., it may be inconsequential that the Kaimal correction outperforms the posterior correction for measurements that were fairly good to begin with. Meanwhile, the difference in $\sigma_{w}$ was large, though it is unclear if the posterior correction makes this significantly better or worse (Table 3). This lack of clarity means the askew manipulation cannot be used to validate or falsify the posterior correction. This is not surprising, because the posterior correction was estimated without data from or knowledge of such a unique manipulation, and, as it is, much of the posterior correction contains a large uncertainty (Fig. 7a). Though the posterior correction is too uncertain to explain the askew manipulation, this does not mean our estimates of $H+\mathrm{LE}$ at various field sites are flawed because these estimates account for the fact that much of the posterior is uncertain. We expect that expanding our Bayesian analysis to include data from more manipulations, e.g., the askew example, would further constrain the regions of uncertainty found in the current posterior correction.

Our results using the posterior correction (Fig. 10) show that there is still unexplained residual error, though we expect some of this to be reduced with our suggestions above. While Horst et al. (2015) showed that to a first order that transducer shadowing is a function of the longitude and latitude of the instantaneous wind, the impact of other covariates such as wind velocity and turbulence may need to be considered. An advantage of performing our analysis in a Bayesian framework is that the model can be expanded to incorporate the effects of these covariates.

And finally, our posterior correction and methodology should be compared to other independent analysis of sonic anemometer shadowing such as wind tunnel data (Horst et al., 2015) or an independent Doppler lidar system (Sathe et al., 2011). Care should be taken when incorporating these results, as anemometers could respond differently under laminar flow in a wind tunnel versus under turbulent field conditions. Regardless, a key to resolving this problem will be to embrace new technologies, new experimental designs, and new analyses.

\section{Conclusions}

The non-orthogonal CSAT3 sonic anemometer produces different results (Fig. 2) when it is mounted horizontally instead of vertically (Fig. 1). Assuming that the primary source of this error is shadowing across the various transducers, a Bayesian model can estimate a posterior correction (Fig. 8) that ultimately makes measurements from vertically and horizontally mounted anemometers most similar (Fig. 10). Even when taking into account the uncertainty of the posterior 
correction (Fig. 7), the increases in vertical wind velocity and sensible heat flux measurements are significantly larger and are approximately twice the magnitude of the Kaimal correction (Fig. 11). When this posterior correction is applied to various eddy-covariance sites across North America, the turbulent components of the ecosystem energy balance (sensible plus latent heat flux) increased between 8.1 and $11.6 \%$, with an average $95 \%$ confidence that this increase was between 6.1 and $13.8 \%$ (Table 2). Considering this is the most common sonic anemometer in the AmeriFlux network and is found in all the regional networks that comprise FLUXNET, these results have major implications for countless studies that use the eddy-covariance technique to measure terrestrial-atmospheric exchange of mass and energy.

\section{Data availability}

The data and source code are available at: https://doi.org/10. 2737/RDS-2016-0037. 


\section{Appendix A}

\section{A1 Univariate conditional posterior distribution functions for Gibbs sampling}

For the univariate conditional posterior distribution functions there is a distinction between independent grid points and those linked together through symmetry. In the case of the former, these functions can be evaluated for each unique grid point, $g$, and for each transducer pair, $t$. In the case of the latter, $g$ and $t$ refer to the sets of all grid points and transducers that share the same unique state variable for their shadow correction, and these functions can be applied to each of these unique sets.

First, using Bayes' theorem, the joint posterior distribution of the model parameters can be expressed as being proportional to the product of the likelihood of the data and the joint prior distribution of the model parameters (Eq. A1).

$p\left(\widetilde{\sigma}_{\mathrm{C}}, \alpha_{\mathrm{T}}, \varepsilon \mid \sigma_{\mathrm{C}}\right) \propto p\left(\sigma_{\mathrm{C}} \mid \widetilde{\sigma}_{\mathrm{C}}, \alpha_{\mathrm{T}}, \varepsilon\right) p\left(\widetilde{\sigma}_{\mathrm{C}}, \alpha_{\mathrm{T}}, \varepsilon\right)$

Because the prior distributions for three model parameters are independent, the joint prior distribution can be written as the product of the individual probabilities (Eq. A2).

$p\left(\widetilde{\sigma}_{\mathrm{C}}, \alpha_{\mathrm{T}}, \varepsilon \mid \sigma_{\mathrm{C}}\right) \propto p\left(\sigma_{\mathrm{C}} \mid \widetilde{\sigma}_{\mathrm{C}}, \alpha_{\mathrm{T}}, \varepsilon\right) p\left(\widetilde{\sigma}_{\mathrm{C}}\right) p\left(\alpha_{\mathrm{T}}\right) p(\varepsilon)$

The likelihood of the data is normally distributed (Eq. A3).

$p\left(\sigma_{\mathrm{C}} \mid \widetilde{\sigma}_{\mathrm{C}}, \alpha_{\mathrm{T}}, \varepsilon\right)=\frac{1}{\sqrt{2 \pi} \varepsilon} e^{\left(-\frac{1}{2 \varepsilon^{2}}\left(\sigma_{\mathrm{C}}-\hat{\sigma}_{\mathrm{C}}\right)^{2}\right)}$

Because $\hat{\sigma}_{\mathrm{C}}$ is a function of both $\widetilde{\sigma}_{\mathrm{C}}$ and $\alpha_{\mathrm{T}}$, the likelihood is indeed a function of all three model parameters. The individual prior distributions for $\widetilde{\sigma}_{\mathrm{C}}, \alpha_{\mathrm{T}}$, and $\varepsilon$ are uniformly (Eq. A4), normally (Eq. A5), and gamma (Eq. A6) distributed, respectively.

$p\left(\widetilde{\sigma}_{\mathrm{C}}\right)= \begin{cases}\frac{1}{\max \left(U_{\mathrm{C}}\right)}, & 0 \leq \widetilde{\sigma}_{\mathrm{C}} \leq \max \left(U_{\mathrm{C}}\right) \\ 0, & \text { otherwise }\end{cases}$

$p\left(\alpha_{\mathrm{T}_{t, g}}\right)=\frac{1}{\sqrt{2 \pi}(0.1)} e^{\left(-\frac{1}{2(0.1)^{2}}\left(\alpha_{\mathrm{T}_{t, g}}-P(t, g)\right)^{2}\right)}$

$p(\varepsilon)=\hat{b} e^{-\dot{b} \varepsilon}$

Gibbs sampling for each model parameter is based on the univariate conditional posterior distribution, which assumes that all other model parameters plus the data are given (in the case of sampling within a multidimensional array, all other parameters within that array are given except the one at the index being evaluated). For $\widetilde{\sigma}_{C}$ the univariate conditional posterior distribution can be expressed as a form of Bayes' theorem (Eq. A7).

$p\left(\widetilde{\sigma}_{\mathrm{C}_{c, f}} \mid{\underline{\widetilde{\sigma}_{\mathrm{C}}}}_{-c, f}, \underline{\alpha_{\mathrm{T}}}, \varepsilon, \underline{\sigma_{\mathrm{C}}}\right)=\frac{p\left(\underline{{\widetilde{\sigma_{\mathrm{C}}}}}, \underline{\alpha_{\mathrm{T}}}, \varepsilon \mid \underline{\sigma_{\mathrm{C}}}\right) p\left(\underline{\sigma_{\mathrm{C}}}\right)}{p\left({\underline{\widetilde{\sigma}_{\mathrm{C}}}}_{-f, c}, \underline{\alpha_{\mathrm{T}}}, \varepsilon, \underline{\sigma_{\mathrm{C}}}\right)}$
The underbar denotes all elements within a multidimensional array, while the notation $\underline{\widetilde{\sigma}}_{-c, f}$ means all elements of $\widetilde{\sigma}_{\mathrm{C}}$ except for $\widetilde{\sigma}_{\mathrm{C}_{c, f}}$. On right side of Eq. (A7), both the second term in the numerator and the denominator are assumed given and can be omitted if the equal sign is changed to a proportional sign. The first term in the numerator, $p\left({\widetilde{\sigma_{\mathrm{C}}}}, \alpha_{\mathrm{T}}, \varepsilon \mid \underline{\sigma_{\mathrm{C}}}\right)$, is the joint posterior distribution summed across all parameters (Eq. A8).

$$
\begin{aligned}
& p\left(\underline{\widetilde{\sigma}_{\mathrm{C}}}, \underline{\alpha_{\mathrm{T}}}, \varepsilon \mid \underline{\sigma_{\mathrm{C}}}\right) \propto \prod_{c=1}^{3} \prod_{f=1}^{F} \\
& \left\{\left[\prod_{i=1}^{I} p\left(\sigma_{\mathrm{C}_{c, f, i}} \mid \widetilde{\sigma}_{\mathrm{C}_{c, f}}, \alpha_{\mathrm{T}}, \varepsilon\right)\right] p\left(\widetilde{\sigma}_{\mathrm{C}_{c, f}}\right)\right\} \\
& \prod_{t=1}^{3} \prod_{g=1}^{G} p\left(\alpha_{\mathrm{T}_{t, g}}\right) p(\varepsilon)
\end{aligned}
$$

Assuming that all but $\widetilde{\sigma}_{\mathrm{C}_{c, f}}$ are given plus requiring that the proposed value for $\widetilde{\sigma}_{C_{c, f}}$ is within the valid range (i.e., $p\left(\widetilde{\sigma}_{\mathrm{C}_{c, f}}\right)$ is constant and can be omitted), Eq. (A7) simplifies to Eq. (A9).

$p\left(\widetilde{\sigma}_{\mathrm{C}_{c, f}}{\underline{\widetilde{\sigma}_{\mathrm{C}}}}_{-c, f}, \underline{\alpha_{\mathrm{T}}}, \varepsilon, \underline{\sigma_{\mathrm{C}}}\right) \propto$

$\prod_{i=1}^{I} p\left(\sigma_{\mathrm{C}_{c, f, i}} \mid \widetilde{\sigma}_{\mathrm{C}_{c, f}}, \alpha_{\mathrm{T}}, \varepsilon\right)$

Substituting in the likelihood from Eq. (A3) and simplifying gives the univariate conditional posterior distribution for $\widetilde{\sigma}_{\mathrm{C}_{c, f}}$ (Eq. A10).

$p\left(\widetilde{\sigma}_{\mathrm{C}_{c, f}} \mid{\underline{\widetilde{\sigma}_{\mathrm{C}}}}_{-c, f}, \underline{\alpha_{\mathrm{T}}}, \varepsilon, \underline{\sigma_{\mathrm{C}}}\right) \propto$

$e^{\left(-\frac{1}{2 \varepsilon^{2}} \sum_{i=1}^{I}\left(\sigma_{C_{c, f, i}}-\hat{\sigma}_{C_{c, f, i}}\right)^{2}\right)}$

The univariate conditional posterior distribution for $\alpha_{\mathrm{T}}$ can be expressed as Bayes' theorem (Eq. A11).

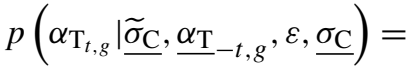

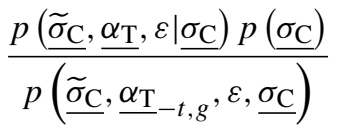

Again, only the first term in the numerator must be evaluated while assuming that all but $\alpha_{\mathrm{T}_{t, g}}$ are given (Eq. A12).

$$
\begin{aligned}
& p\left(\alpha_{\mathrm{T}_{t, g}} \mid \underline{\widetilde{\sigma}_{\mathrm{C}}}, \underline{\alpha}_{\mathrm{T}}-t, g\right. \\
& \left.\prod_{c=1}, \varepsilon, \underline{\sigma_{\mathrm{C}}}\right) \propto \\
& \prod_{f=1}^{3} \prod_{i=1}^{F} p\left(\sigma_{\mathrm{C}_{c, f, i}} \mid \widetilde{\sigma}_{\mathrm{C}_{c, f}}, \alpha_{\mathrm{T}}, \varepsilon\right) p\left(\alpha_{\mathrm{T}_{t, g}}\right)
\end{aligned}
$$

Substituting in both the likelihood of the data (Eq. A3) and the prior distribution for $\alpha_{\mathrm{T}_{t, g}}$ (Eq. A5) and simplifying yields the univariate conditional posterior distribution for $\alpha_{\mathrm{T}_{t, g}}$ (Eq. A13). 


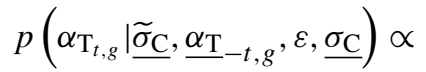

$$
\begin{aligned}
& e^{\left(-\frac{1}{2 \varepsilon^{2}} \sum_{c=1}^{3} \sum_{f=1}^{F} \sum_{i=1}^{I}\left(\sigma_{\mathrm{C}_{c, f, i}}-\hat{\sigma}_{\mathrm{C}_{c, f, i}}\right)^{2}-\frac{1}{2(0.1)^{2}}\left(\alpha_{\mathrm{T}_{t, g}}-P(t, g)\right)^{2}\right)}
\end{aligned}
$$

An important issue is that $\hat{\sigma}_{\mathrm{C}}$ is a function of $\alpha_{\mathrm{T}}$ and must be evaluated for every proposed change to the 3 -D correction. This is computationally intensive and causes a bottleneck in the analysis. Finally, the univariate conditional posterior distribution for $\varepsilon$ can be expressed as Bayes' theorem (Eq. A14).

$$
p\left(\varepsilon \mid \underline{\widetilde{\sigma}_{\mathrm{C}}}, \underline{\alpha_{\mathrm{T}}}, \underline{\sigma_{\mathrm{C}}}\right)=\frac{p\left(\underline{\widetilde{\sigma}_{\mathrm{C}}}, \underline{\alpha_{\mathrm{T}}}, \varepsilon \mid \underline{\sigma_{\mathrm{C}}}\right) p\left(\underline{\sigma_{\mathrm{C}}}\right)}{p\left(\underline{\widetilde{\sigma}_{\mathrm{C}}}, \underline{\alpha_{\mathrm{T}}}, \underline{\sigma_{\mathrm{C}}}\right)}
$$

Only the first term in the numerator must be evaluated while assuming that all but $\varepsilon$ are given (Eq. A15).

$$
\begin{array}{r}
p\left(\varepsilon \mid \underline{\widetilde{\sigma}_{\mathrm{C}}}, \underline{\alpha_{\mathrm{T}}}, \underline{\sigma_{\mathrm{C}}}\right) \propto \prod_{c=1}^{3} \prod_{f=1}^{F} \prod_{i=1}^{I} \\
p\left(\sigma_{\mathrm{C}_{c, f, i}} \mid \widetilde{\sigma}_{\mathrm{C}_{c, f}}, \alpha_{\mathrm{T}}, \varepsilon\right) p(\varepsilon)
\end{array}
$$

Substituting in the likelihood from Eq. (A3) and simplifying yields the univariate conditional posterior distribution for $\varepsilon$ (Eq. A16)

$$
\begin{aligned}
& p\left(\varepsilon \mid \underline{\widetilde{\sigma}_{\mathrm{C}}}, \underline{\alpha_{\mathrm{T}}}, \underline{\left.\sigma_{\mathrm{C}}\right) \propto}\right. \\
& \varepsilon^{-3 \mathrm{FI}} e^{\left(-\frac{1}{2 \varepsilon^{2}} \sum_{c=1}^{3} \sum_{f=1}^{F} \sum_{i=1}^{I}\left(\sigma_{\mathrm{C}_{c, f, i}}-\hat{\sigma}_{\mathrm{C}_{c, f, i}}\right)^{2}-\dot{b} \varepsilon\right)}
\end{aligned}
$$

\section{A2 Instability in the $w$ correction for near-equatorial winds}

For a CSAT3, the amount of correction applied to the vertical wind velocity - expressed as the individual corrections $\alpha_{A}(\lambda, \varphi), \alpha_{B}(\lambda, \varphi)$, and $\alpha_{C}(\lambda, \varphi)$ for the three transducer pairs $A, B$, and $C$ as functions of longitude, $\lambda$, and latitude, $\varphi-$ is

$$
\begin{aligned}
\frac{\boldsymbol{w}_{\text {corrected }}}{\boldsymbol{w}_{\text {uncorrected }}} & =\frac{2}{3 \sqrt{3}}\left[\left(-\frac{\cos \lambda}{2 \tan \varphi}+\frac{\sqrt{3}}{2}\right) \alpha_{A}(\lambda, \varphi)\right. \\
& +\left(\frac{\cos \lambda+\sqrt{3} \sin \lambda}{4 \tan \varphi}+\frac{\sqrt{3}}{2}\right) \alpha_{B}(\lambda, \varphi) \\
& \left.+\left(\frac{\cos \lambda-\sqrt{3} \sin \lambda}{4 \tan \varphi}+\frac{\sqrt{3}}{2}\right) \alpha_{C}(\lambda, \varphi)\right]
\end{aligned}
$$

If the individual corrections for the three transducer pairs never approach 0 or $\pm \infty$, which is a safe assumption considering they are always around 1 (Figs. 3a, c, e and 9a, c, e), the limit of this as the latitude approaches the Equator is

$$
\begin{aligned}
\lim _{\varphi \rightarrow 0} \frac{\boldsymbol{w}_{\text {corrected }}}{\boldsymbol{w}_{\text {uncorrected }}} & =\frac{1}{3}\left(\alpha_{A}(\lambda, \varphi)+\alpha_{B}(\lambda, \varphi)+\alpha_{C}(\lambda, \varphi)\right) \\
& +\frac{2}{3 \sqrt{3}}\left[\left(-\frac{\cos \lambda}{2}\right) \alpha_{A}(\lambda, \varphi)\right. \\
& +\left(\frac{\cos \lambda+\sqrt{3} \sin \lambda}{4}\right) \alpha_{B}(\lambda, \varphi) \\
& \left.+\left(\frac{\cos \lambda-\sqrt{3} \sin \lambda}{4}\right) \alpha_{C}(\lambda, \varphi)\right] \lim _{\varphi \rightarrow 0} \frac{1}{\tan \varphi} .
\end{aligned}
$$

This approaches $\pm \infty$ unless the terms associated with the limit of the tangent exactly cancel. This is achieved if $\alpha_{A}\left(\lambda, 0^{\circ}\right)=\alpha_{B}\left(\lambda, 0^{\circ}\right)=\alpha_{C}\left(\lambda, 0^{\circ}\right)$, which includes the special case where $\alpha_{A}\left(\lambda, 0^{\circ}\right)=\alpha_{B}\left(\lambda, 0^{\circ}\right)=\alpha_{C}\left(\lambda, 0^{\circ}\right)=1$. Based on our assumptions of symmetry with the CSAT3, $\quad \alpha_{B}(\lambda, \varphi)=\alpha_{A}\left(60^{\circ}-\lambda,-\varphi\right) \quad$ and $\quad \alpha_{C}(\lambda, \varphi)=$ $\alpha_{A}\left(60^{\circ}+\lambda,-\varphi\right)$. Therefore, the $w$ correction for nearequatorial winds is unstable unless

$$
\begin{aligned}
\alpha_{A}\left(\lambda, 0^{\circ}\right) & =\frac{1+\sqrt{3} \tan \lambda}{2} \alpha_{A}\left(60^{\circ}-\lambda, 0^{\circ}\right) \\
& +\frac{1-\sqrt{3} \tan \lambda}{2} \alpha_{A}\left(60^{\circ}+\lambda, 0^{\circ}\right) .
\end{aligned}
$$

This is satisfied by $\lambda=30,90,150,210,270$, and $330^{\circ}$. Equation (A19) shows that if the weighted average of $\alpha_{A}\left(60^{\circ}-\lambda,-\varphi\right)$ and $\alpha_{A}\left(60^{\circ}+\lambda,-\varphi\right)$ cancel $\alpha_{A}\left(\lambda, 0^{\circ}\right)$ then the correction will be stable. This cannot be achieved if the correction $\alpha_{A}\left(\lambda, 0^{\circ}\right)$ is monotonic within $0^{\circ} \leq \lambda \leq 90^{\circ}$. Because the $\boldsymbol{w}$ correction is symmetric every $30^{\circ}$, any solution besides $\lambda=30,90,150,210,270$, and $330^{\circ}$ will be mirrored 12 times. 


\section{The Supplement related to this article is available online at doi:10.5194/amt-9-5933-2016-supplement.}

Acknowledgements. We thank Jorge Ramirez, Susan Howe, Mario Bretfeld, Kelly Elder, Banning Starr, Bill Kustas, and Joe Alfieri for providing data from their unique field sites. We especially thank Ben Bird for his comments and countless hours of statistical advice in developing the Bayesian model. We thank Jay Ham for his generous assistance in conducting the field validation experiment. We thank Bob Hall and the two anonymous reviewers, whose thoughts and comments improved this manuscript. This study was funded by the US Forest Service, the Wyoming Water Development Commission, the USGS, the NSF (awards EPS-1208909 and EAR-0444053), and the DOD Army Research Office (W911NF05-1-0558 and W911NF-05-1-0126).

Edited by: L. Bianco

Reviewed by: three anonymous referees

\section{References}

Anderson, R. G. and Wang, D.: Energy budget closure observed in paired eddy covariance towers with increased and continuous daily turbulence, Agr. Forest Meteorol., 184, 204-209, doi:10.1016/j.agrformet.2013.09.012, 2014.

Baldocchi, D. D.: Assessing the eddy covariance technique for evaluating carbon dioxide exchange rates of ecosystems: past, present and future, Glob. Change Biol., 9, 479-492, doi:10.1046/j.1365-2486.2003.00629.x, 2003.

Barr, A. G., Morgenstern, K., Black, T. A., McCaughey, J. H., and Nesic, Z.: Surface energy balance closure by the eddy-covariance method above three boreal forest stands and implications for the measurement of the $\mathrm{CO}_{2}$ flux, Agr. Forest Meteorol., 140, 322337, doi:10.1016/j.agrformet.2006.08.007, 2006.

Barrett, E. W. and Suomi, V. E.: Preliminary report on temperature measurement by sonic means, J. Meteorol., 6, 273-276, doi:10.1175/1520-0469(1949)006<0273:PROTMB>2.0.CO;2, 1949.

Bayes, M. and Price, M.: An Essay towards solving a problem in the doctrine of chances. By the Late Rev. Mr. Bayes, F. R. S. Communicated by Mr. Price, in a letter to John Canton, A. M. F. R. S, Phil. Trans., 53, 370-418, doi:10.1098/rstl.1763.0053, 1763.

Biederman, J. A., Harpold, A. A., Gochis, D. J., Ewers, B. E., Reed, D. E., Papuga, S. A., and Brooks, P. D.: Increased evaporation following widespread tree mortality limits streamflow response, Water Resour. Res., 50, 5395-5409, doi:10.1002/2013wr014994, 2014.

Buks, E., Schuster, R., Heiblum, M., Mahalu, D., and Umansky, V.: Dephasing in electron interference by a "which-path" detector, Nature, 391, 871-874, 1998.

Dellwik, E., Sjöholm, M., and Mann, J.: An evaluation of the WindEye wind lidar, DTU Wind Energy, Report, 2015.

Fisher, S. G. and Likens, G. E.: Energy flow in Bear Brook, New Hampshire: An integrative approach to stream ecosystem metabolism, Ecol. Monogr., 43, 421-439, doi:10.2307/1942301, 1973.

Foken, T.: The energy balance closure problem: An overview, Ecol. Appl., 18, 1351-1367, doi:10.1890/06-0922.1, 2008.

Frank, J. M., Massman, W. J., and Ewers, B. E.: Underestimates of sensible heat flux due to vertical velocity measurement errors in non-orthogonal sonic anemometers, Agr. Forest Meteorol., 171172, 72-81, doi:10.1016/j.agrformet.2012.11.005, 2013.

Frank, J. M., Massman, W. J., Ewers, B. E., Huckaby, L. S., and Negrón, J. F.: Ecosystem $\mathrm{CO}_{2} / \mathrm{H}_{2} \mathrm{O}$ fluxes are explained by hydraulically limited gas exchange during tree mortality from spruce bark beetles, J. Geophys. Res.-Biogeo., 119, 1195-1215, doi:10.1002/2013jg002597, 2014.

Frank, J. M., Massman, W. J., Swiatek, E., Zimmerman, H. A., and Ewers, B. E.: All sonic anemometers need to correct for transducer and structural shadowing in their velocity measurements, J. Atmos. Ocean. Tech., 33, 149-167, doi:10.1175/jtech-d-150171.1, 2016.

Franssen, H. J. H., Stockli, R., Lehner, I., Rotenberg, E., and Seneviratne, S. I.: Energy balance closure of eddy-covariance data: A multisite analysis for European FLUXNET stations, Agr. Forest Meteorol., 150, 1553-1567, doi:10.1016/j.agrformet.2010.08.005, 2010.

Friebel, H. C., Herrington, T. O., and Benilov, A. Y.: Evaluation of the flow distortion around the Campbell Scientific CSAT3 sonic anemometer relative to incident wind direction, J. Atmos. Ocean. Tech., 26, 582-592, doi:10.1175/2008jtecho550.1, 2009.

Gelman, A., Carlin, J. B., Stern, H. S., Dunson, D. B., Vehtari, A., and Rubin, D. B.: Bayesian data analysis, 3rd Edn., CRC Press, Boca Raton, FL, 661 pp., 2014.

Heilman, J. L., McInnes, K. J., Kjelgaard, J. F., Keith Owens, M., and Schwinning, S.: Energy balance and water use in a subtropical karst woodland on the Edwards Plateau, Texas, J. Hydrology, 373, 426-435, doi:10.1016/j.jhydrol.2009.05.007, 2009.

Hogstrom, U. and Smedman, A. S.: Accuracy of sonic anemometers: Laminar wind-tunnel calibrations compared to atmospheric in situ calibrations against a reference instrument, Bound.-Lay. Meteorol., 111, 33-54, doi:10.1023/b:boun.0000011000.05248.47, 2004.

Horst, T. W., Semmer, S. R., and Maclean, G.: Correction of a nonorthogonal, three-component sonic anemometer for flow distortion by transducer shadowing, Bound.-Lay. Meteorol., 155, 371395, doi:10.1007/s10546-015-0010-3, 2015.

Kaimal, J. C.: Sonic anemometer measurement of atmospheric turbulence, Proceedings of the Dynamic Flow Conference 1978 on Dynamic Measurements in Unsteady Flows, Johns Hopkins University, Baltimore, USA, 18-21 September 1978, 551-565, 1979.

Kaimal, J. C. and Businger, J. A.: A continuous wave sonic anemometer-thermometer, J. Appl. Meteorol., 2, 156-164, doi:10.1175/1520-0450(1963)002<0156:acwsat>2.0.co;2, 1963.

Kochendorfer, J., Meyers, T. P., Frank, J. M., Massman, W. J., and Heuer, M. W.: How well can we measure the vertical wind speed? Implications for fluxes of energy and mass, Bound.-Lay. Meteorol., 145, 383-398, doi:10.1007/s10546-012-9738-1, 2012.

Kruschke, J.: Doing Bayesian data analysis: A tutorial introduction with R and BUGS, Academic Press, 2010.

Lee, X., Finnigan, J. J., and Paw U, K. T.: Coordinate system and flux bias error, in: Handbook of micrometeorology, edited by: 
Lee, X., Massman, W. J., and Law, B. E., Kluwer Academic Publishers, Dordrecht, the Netherlands, 33-66, 2004.

Leuning, R., van Gorsel, E., Massman, W. J., and Isaac, P. R.: Reflections on the surface energy imbalance problem, Agr. Forest Meteorol., 156, 65-74, doi:10.1016/j.agrformet.2011.12.002, 2012.

Lu, Y., Ye, K., Mathur, A. K., Hui, S., Fuerst, T. P., and Genant, H. K.: Comparative calibration without a gold standard, Stat. Med., 16, 1889-1905, doi:10.1002/(sici)10970258(19970830)16:16<1889::aid-sim607>3.0.co;2-v, 1997.

MacLean, W. R.: Absolute Measurement of Sound Without a Primary Standard, J. Acoust. Soc. Am., 12, 140-146, doi:10.1121/1.1916085, 1940.

Massman, W. J. and Lee, X.: Eddy covariance flux corrections and uncertainties in long-term studies of carbon and energy exchanges, Agr. Forest Meteorol., 113, 121-144, doi:10.1016/s0168-1923(02)00105-3, 2002.

Monnier, T., Seydou, D., Godin, N., and Zhang, F.: Primary calibration of acoustic emission sensors by the method of reciprocity, theoretical and experimental considerations, J. Acoust. Emission, 30, 152-166, 2012.

Nakai, T. and Shimoyama, K.: Ultrasonic anemometer angle of attack errors under turbulent conditions, Agr. Forest Meteorol., 162-163, 14-26, doi:10.1016/j.agrformet.2012.04.004, 2012.

Nakai, T., Iwata, H., Harazono, Y., and Ueyama, M.: An inter-comparison between Gill and Campbell sonic anemometers, Agr. Forest Meteorol., 195-196, 123-131, doi:10.1016/j.agrformet.2014.05.005, 2014.

Odum, H. T.: Trophic structure and productivity of Silver Springs, Florida, Ecol. Monogr., 27, 55-112, doi:10.2307/1948571, 1957.

Oliphant, A. J., Grimmond, C. S. B., Zutter, H. N., Schmid, H. P., Su, H. B., Scott, S. L., Offerle, B., Randolph, J. C., and Ehman, J.: Heat storage and energy balance fluxes for a temperate deciduous forest, Agr. Forest Meteorol., 126, 185-201, doi:10.1016/j.agrformet.2004.07.003, 2004.

R Core Team: R: A language and environment for statistical computing. R Foundation for Statistical Computing, Vienna, Austria, availabele at: https://www.R-project.org/ (last access: 21 October 2015), 2015.

Reba, M. L., Pomeroy, J., Marks, D., and Link, T. E.: Estimating surface sublimation losses from snowpacks in a mountain catchment using eddy covariance and turbulent transfer calculations, Hydrol. Process., 26, 3699-3711, doi:10.1002/hyp.8372, 2012.

RStudio Team: RStudio: Integrated Development for R. RStudio, Inc., Boston, MA, available at: http://www.rstudio.com/ (last access: 21 October 2015), 2015.

Sathe, A., Mann, J., Gottschall, J., and Courtney, M. S.: Can wind Lidars measure turbulence?, J. Atmos. Ocean. Tech., 28, 853868, doi:10.1175/jtech-d-10-05004.1, 2011.

Scott, R. L., Edwards, E. A., Shuttleworth, W. J., Huxman, T. E., Watts, C., and Goodrich, D. C.: Interannual and seasonal variation in fluxes of water and carbon dioxide from a riparian woodland ecosystem, Agr. Forest Meteorol., 122, 65-84, doi:10.1016/j.agrformet.2003.09.001, 2004.
Stoy, P. C., Mauder, M., Foken, T., Marcolla, B., Boegh, E., Ibrom, A., Arain, M. A., Arneth, A., Aurela, M., Bernhofer, C., Cescatti, A., Dellwik, E., Duce, P., Gianelle, D., van Gorsel, E., Kiely, G., Knohl, A., Margolis, H., McCaughey, H., Merbold, L., Montagnani, L., Papale, D., Reichstein, M., Saunders, M., Serrano-Ortiz, P., Sottocornola, M., Spano, D., Vaccari, F., and Varlagin, A.: A data-driven analysis of energy balance closure across FLUXNET research sites: The role of landscape scale heterogeneity, Agr. Forest Meteorol., 171-172, 137-152, doi:10.1016/j.agrformet.2012.11.004, 2013.

Teal, J. M.: Energy flow in the salt marsh ecosystem of Georgia, Ecology, 43, 614-624, doi:10.2307/1933451, 1962.

Twine, T. E., Kustas, W. P., Norman, J. M., Cook, D. R., Houser, P. R., Meyers, T. P., Prueger, J. H., Starks, P. J., and Wesely, M. L.: Correcting eddy-covariance flux underestimates over a grassland, Agr. Forest Meteorol., 103, 279-300, doi:10.1016/s01681923(00)00123-4, 2000.

van der Molen, M. K., Gash, J. H. C., and Elbers, J. A.: Sonic anemometer (co)sine response and flux measurement - II. The effect of introducing an angle of attack dependent calibration, Agr. Forest Meteorol., 122, 95-109, doi:10.1016/j.agrformet.2003.09.003, 2004.

Wahba, G.: Spline interpolation and smoothing on the sphere, SIAM J. Sci. Stat. Comp., 2, 5-16, doi:10.1137/0902002, 1981.

Wang, R., Zhang, Q., Zhao, H., Wang, H., and Wang, C.: Analysis of the surface energy closure for a site in the Gobi Desert in Northwest China, Acta Meteorol. Sin., 26, 250-259, doi:10.1007/s13351-012-0210-4, 2012.

Webb, E. K., Pearman, G. I., and Leuning, R.: Correction of flux measurements for density effects due to heat and water vapour transfer, Q. J. Roy. Meteor. Soc., 106, 85-100, doi:10.1256/smsqj.44706, 1980.

Welch, C. M., Stoy, P. C., Rains, F. A., Johnson, A. V., and McGlynn, B. L.: The impacts of mountain pine beetle disturbance on the energy balance of snow during the melt period, Hydrol. Process., 30, 588-602, doi:10.1002/hyp.10638, 2015.

Wilson, K., Goldstein, A., Falge, E., Aubinet, M., Baldocchi, D., Berbigier, P., Bernhofer, C., Ceulemans, R., Dolman, H., Field, C., Grelle, A., Ibrom, A., Law, B. E., Kowalski, A., Meyers, T., Moncrieff, J., Monson, R., Oechel, W., Tenhunen, J., Valentini, R., and Verma, S.: Energy balance closure at FLUXNET sites, Agr. Forest Meteorol., 113, 223-243, doi:10.1016/s01681923(02)00109-0, 2002.

Wood, S. N.: Generalized additive models: an introduction with R, Chapman and Hall/CRC, 2006.

Wyngaard, J. C. and Zhang, S.-F.: Transducer-shadow effects on turbulence spectra measured by sonic anemometers, J. Atmos. Ocean. Tech., 2, 548-558, doi:10.1175/15200426(1985)002<0548:tseots>2.0.co;2, 1985.

Yahaya, S. and Frangi, J. P.: Cup anemometer response to the wind turbulence-measurement of the horizontal wind variance, Ann. Geophys., 22, 3363-3374, doi:10.5194/angeo-22-33632004, 2004. 